\title{
Review
}

\section{Red Meat and Colorectal Cancer: A Quantitative Update on the State of the Epidemiologic Science}

\author{
Dominik D. Alexander, PhD, MSPH, Douglas L. Weed, MD, PhD, Paula E. Miller, MPH, Muhima A. Mohamed, MPH \\ EpidStat Institute, Evergreen, Colorado (D.D.A.); EpidStat Institute, Ann Arbor, Michigan (D.D.A., P.E.M.); DLW Consulting \\ Services, Salt Lake City, Utah (D.L.W.); Doctors Without Borders, Bellevue, Washington (M.A.M.)
}

Key words: cancer, epidemiology, meta-analysis, colorectal cancer, cohort studies

\begin{abstract}
The potential relationship between red meat consumption and colorectal cancer (CRC) has been the subject of scientific debate. Given the high degree of resulting uncertainty, our objective was to update the state of the science by conducting a systematic quantitative assessment of the epidemiologic literature. Specifically, we updated and expanded our previous meta-analysis by integrating data from new prospective cohort studies and conducting a broader evaluation of the relative risk estimates by specific intake categories. Data from 27 independent prospective cohort studies were meta-analyzed using random-effects models, and sources of potential heterogeneity were examined through subgroup and sensitivity analyses. In addition, a comprehensive evaluation of potential dose-response patterns was conducted. In the meta-analysis of all cohorts, a weakly elevated summary relative risk was observed (1.11, 95\% CI: 1.03-1.19); however, statistically significant heterogeneity was present. In general, summary associations were attenuated (closer to the null and less heterogeneous) in models that isolated fresh red meat (from processed meat), adjusted for more relevant factors, analyzed women only, and were conducted in countries outside of the United States. Furthermore, no clear patterns of dose-response were apparent. In conclusion, the state of the epidemiologic science on red meat consumption and CRC is best described in terms of weak associations, heterogeneity, an inability to disentangle effects from other dietary and lifestyle factors, lack of a clear dose-response effect, and weakening evidence over time.
\end{abstract}

\section{Key Teaching Points:}

- The role of red meat consumption in colorectal cancer risk has been widely contested among the scientific community.

- In the current meta-analysis of red meat intake and colorectal cancer, we comprehensively examined associations by creating numerous sub-group stratifications, conducting extensive sensitivity analyses, and evaluating dose-response using several different methods.

- Overall, all summary associations were weak in magnitude with no clear dose-response patterns.

- Interpretation of findings from epidemiologic studies investigating diet and health outcomes involves numerous methodological considerations, such as accurately measuring food intake, dietary pattern differences across populations, food definitions, outcome classifications, bias and confounding, multicollinearity, biological mechanisms, genetic variation in metabolizing enzymes, and differences in analytical metrics and statistical testing parameters.

\section{INTRODUCTION}

The role of red meat consumption in colorectal cancer (CRC) risk has been widely contested among the scientific community. The uncertainty surrounding this issue contributes to confusion among scientists and dieticians, as well as the general public, when interpreting results from studies, making inferences, and translating these into appropriate dietary recommendations.

The difficulty of interpreting complex epidemiologic evidence transcends the topic of red meat intake and cancer; the broader field of nutritional epidemiology is faced with many

This is an Open Access article. Non-commercial re-use, distribution, and reproduction in any medium, provided the original work is properly attributed, cited, and is not altered, transformed, or built upon in any way, is permitted. The moral rights of the named author(s) have been asserted.

Address correspondence to: Dominik D. Alexander, PhD, MSPH, Principal Epidemiologist, EpidStat Institute, 6539 Jungfrau Way, Evergreen, CO 80439. Email: dalexander@epidstat.com.

Color versions of one or more of the figures in the article can be found online at www.tandfonline.com/uacn.

(C) Dominik D. Alexander, Douglas L. Weed, Paula E. Miller, and Muhima A. Mohamed

Journal of the American College of Nutrition, Vol. 34, No. 6, 521-543 (2015)

Published with license by Taylor \& Francis Group, LLC 
challenges. Interpretation of findings from epidemiologic studies investigating diet and health outcomes involves numerous methodological considerations, such as accurately measuring food intake, dietary pattern differences across populations, food definitions, outcome classifications, bias and confounding, multicollinearity, biological mechanisms, genetic variation in metabolizing enzymes, and differences in analytical metrics and statistical testing parameters [1].

Complicating things further is the fact that studies of dietary factors and cancer often generate weak statistical associations, with relative risks generally ranging between 0.8 and 1.25 . If, on the other hand, there is a strong exposure effect (e.g., relative risks consistently above 2.0 ), sources of uncertainty such as confounding, exposure misclassification, and other biases may not be sufficient to obscure a valid association. However, in the case of weak associations, even modest confounding, moderate exposure and confounder measurement error, and other biases can have a large impact on effect estimates.

The topic of red meat consumption and CRC fits into this methodologically complex paradigm. Several postulated mechanisms have been proposed by which red meat may increase $\mathrm{CRC}$ risk, such as the content of the meat (e.g., heme iron), mutagenic compounds produced by cooking practices (e.g., heterocyclic amines), and gut microbiota composition [2]. However, data from epidemiologic studies of red meat intake and $\mathrm{CRC}$ have not supported clearly the notion that there is an underlying biological mechanism of action. A handful of metaanalyses on this topic have been published over the past decade, and in general, all have produced similar summary estimates (i.e., weak positive associations) based on similar analytical strategies [3-9]. Thus, in the current review, we took a different approach to reviewing the evidence by more thoroughly and transparently examining study-specific associations by intake category. Specifically, we augmented our previous meta-analysis [3] by integrating data from new prospective cohort studies and conducted a broader evaluation of the relative risk estimates by specific intake levels. In addition, we discuss the relevant methodological and analytical factors to consider when interpreting the body of evidence.

\section{METHODS}

\section{Study Identification}

A comprehensive literature search was conducted in MEDLINE and the Cochrane Database to identify articles on red meat consumption and CRC published through 2013. This search supplemented our previous literature search and study inclusion process $[3,10]$. Further, we searched the bibliographies of the World Cancer Research Fund (WCRF)/American Institute for Cancer Research (AICR) report on diet and cancer [11] and their Continuous Update Project (CUP) for CRC, review articles, and meta-analyses pertaining to red meat consumption and CRC in an effort to identify all available literature on this topic.

\section{Study Inclusion}

Peer-reviewed prospective cohort studies and nested casecontrol studies that reported results for the association between red meat consumption and $\mathrm{CRC}$ were included in the metaanalysis. Case-control studies not nested in cohorts, cross-sectional, and ecologic studies were not included. As expected, the definition of red meat varies across studies, and this irregularity is due to researcher distinctions, differences in food composition databases and questionnaires, and geographic variability in meat consumption practices, among other factors. In light of these differences, red meat is commonly defined as beef, pork, lamb, or a combination thereof $[11,12]$. Thus, we included studies that identified meat intake as a composite "red meat" variable or individual red meat items, such as beef, pork, or lamb. Studies that reported data for a broad classification of meat, such as "total meat" categories, which included poultry or fish, were excluded. The definitions of red meat across studies may include some processed red meat items. Further, studies that reported information pertaining to processed meat intake as an independent variable (published previously [13]); constituents of red meat, such as fat or protein from animal sources (published elsewhere [14]); heterocyclic amine exposure; heme iron; cooking practices; or adenomatous polyps were obtained, but these analyses were beyond the scope of the present assessment. Included studies were required to report relative risk estimates (e.g., rate ratios, hazard ratios) and $95 \%$ confidence intervals (CIs) for intake categories of red meat and colorectal, colon, or rectal cancer. Published studies included in this review were written in English. All $(n=38)$ studies meeting the above criteria were obtained, but only studies that represented independent (nonoverlapping) study populations or included extended follow-ups from prior analyses were included in the quantitative assessment $(n=27)$ $[8,15-52]$.

\section{Data Synthesis and Analytical Process}

From each included study, we extracted the following qualitative and quantitative information: author and year of study, geographic study area, the name of the cohort, study size, years of follow-up, red meat definition, method of exposure assessment, intake metric units, analytical comparison of red meat intake metrics, number of exposed cases, relative risk estimates (RRs), 95\% CIs, and the variables that were statistically adjusted for. In addition, a thorough examination of methodological information regarding the potential impact of bias and/ or confounding on the interpretation of each study was conducted. As discussed in greater depth in our previous metaanalysis [3], a review of each article was conducted to 
identify cohorts that may have been analyzed in multiple publications, and this effort was conducted and validated by two researchers.

Combining quantitative data across nutritional epidemiology studies is a challenging endeavor because of the variability in study characteristics, differences in intake metrics, and variation in the type of analyses reported (e.g., red meat, red meat with processed meat, CRC, colon cancer only, women only, etc.). Furthermore, the level of red meat intake can be highly variable across studies. For example, the two lowest categories of intake in one study may be similar to the highest categories of intake in another study [10]. With the understanding that this variability can never be addressed completely, we created numerous meta-analytic models and dose plots to evaluate varying levels of intake. In addition, we conducted several meta-analyses to evaluate other important factors, such as level of adjustment for relevant confounding factors, associations by tumor site and gender, specificity of the red meat variable, and the geographic location of the study.

Statistical analyses were based on comparisons of the highest intake category with the lowest intake category (which may include persons who do not consume red meat). Categorical dose-response analyses using the method proposed by Greenland and Longnecker [53-55] were conducted to estimate the slopes ( $b$ coefficients) from the correlated natural log of the relative risks across intake strata. Each analysis has strengths and limitations. It would be expected that, if an effect was to be observed, it would be found at the extreme intake levels in each study (i.e., high vs low intake); however, intake levels may vary considerably across studies. In a categorical doseresponse analysis, data from all intake categories are used to estimate a linear relationship (or estimates of nonlinear patterns in some cases); however, a foremost assumption is that the slope based on intake levels in one study (e.g., 0-1 serving, 2-3 servings, 4-5 servings) follows a similar linear trajectory in another study that may have higher intake levels (e.g., 0-2 servings, $3-5$ servings, $6-8$ servings, $9+$ servings). Thus, in an effort to harmonize the intake variable of interest (i.e., red meat) among studies, we created intake groupings for grams per day ( $<40 \mathrm{~g}, 40-70 \mathrm{~g}, 71-100 \mathrm{~g},>100 \mathrm{~g}$ ) and servings per week $(0-1,2-4,5-7,>7)$ metrics, as classified in each study. All analytical models were created to serve as complementary analyses, because it would be expected that, if the variable of interest is independently associated with the outcome, consistent findings would be observed across the various analyses. In addition, the RRs and 95\% CIs were plotted for all studies to provide a visual representation of associations across intake levels.

Consumption data were not rescaled across studies, because to do so could introduce another dimension of measurement error. Therefore, all analytical models were created using each study's original data-reporting format, either grams per day or servings per week (rescaled from servings per day where necessary). We did not evaluate individual red meat items because few studies reported associations for such foods, and a high degree of correlation would be expected among meat types (e.g., individuals who consume beef likely consume pork), unless there is a clear geographic, cultural, or food availability distinction. We combined data for beef and pork to estimate a composite average in studies that reported these items individually without reporting values for red meat.

Random-effects models were used to calculate summary relative risk estimates (SRREs), 95\% CIs, and corresponding $P$ values for heterogeneity. The primary meta-analysis models consisted of data from all cohort studies (men and women combined, colon and rectal cancer outcomes), and separate models by sex and anatomic tumor site, as well as sex stratified by tumor site. Additional models included study location, degree of adjustment for confounders, specificity of red meat intake, intake groupings, and publication date. Sensitivity analyses were conducted by evaluating the impact of adding or removing studies based on certain study characteristics. Cumulative meta-analyses were performed to evaluate the modifications in summary effect sizes based on increasing intake levels. If data for men and women or colon and rectum were reported separately in a study, the point estimates and CIs for each sex or each tumor site were included. The presence of publication bias was assessed visually by examining a funnel plot measuring the standard error as a function of effect size, as well as performing Egger's regression method and the Duval and Tweedie imputation method [56]. All statistical analyses were performed using Comprehensive Meta-Analysis (version 2.2.046; Biostat, Englewood, New Jersey, USA; Comprehensive Meta-Analysis).

\section{RESULTS}

Characteristics of all studies included in this assessment are reported in Table 1. Studies varied (often considerably) by several methodological and analytical factors. The majority of studies (based on nonoverlapping cohorts) represent populations in the United States (U.S.) $(n=12)$, followed by Asia (China and Japan) $(n=8)$, various European countries $(n=6)$, and Australia $(n=1)$. The red meat variable included processed meat items in 10 studies; only 17 studies reported data for red meat (independent of processed meat), and 13 of these studies explicitly defined the red meat variable. Fourteen studies reported intake metrics in a grams-per-day format, while 10 studies reported intake levels by servings per day or week. Men and women were analyzed together in many studies; however, several studies reported sex-specific data, and many cohorts were restricted to men only (e.g., Physicians Health Study) or women only (e.g., Iowa Women's Health Study). Similarly, CRC was reported as a combined outcome in many studies, but colon cancer and rectal cancer were reported 


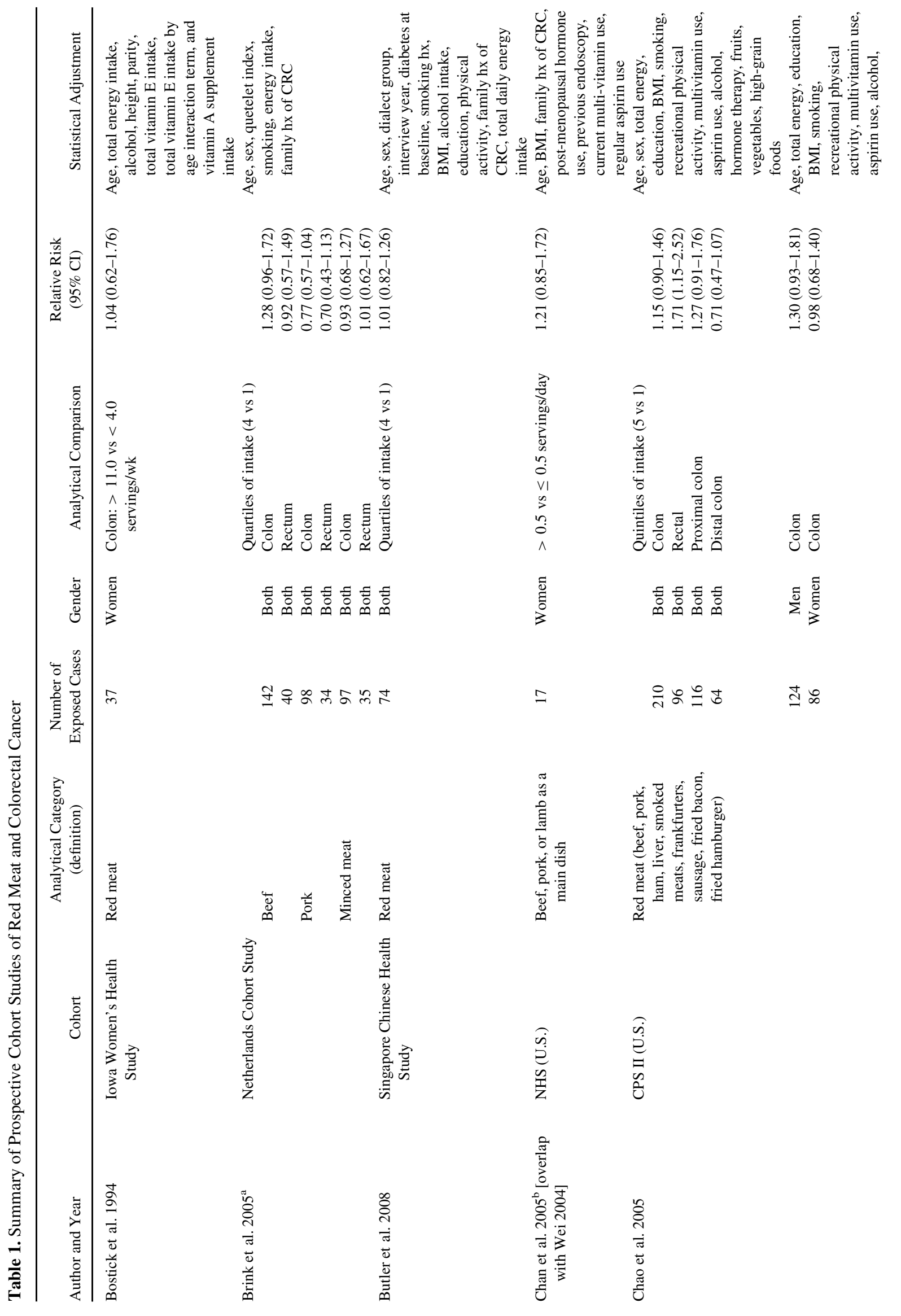






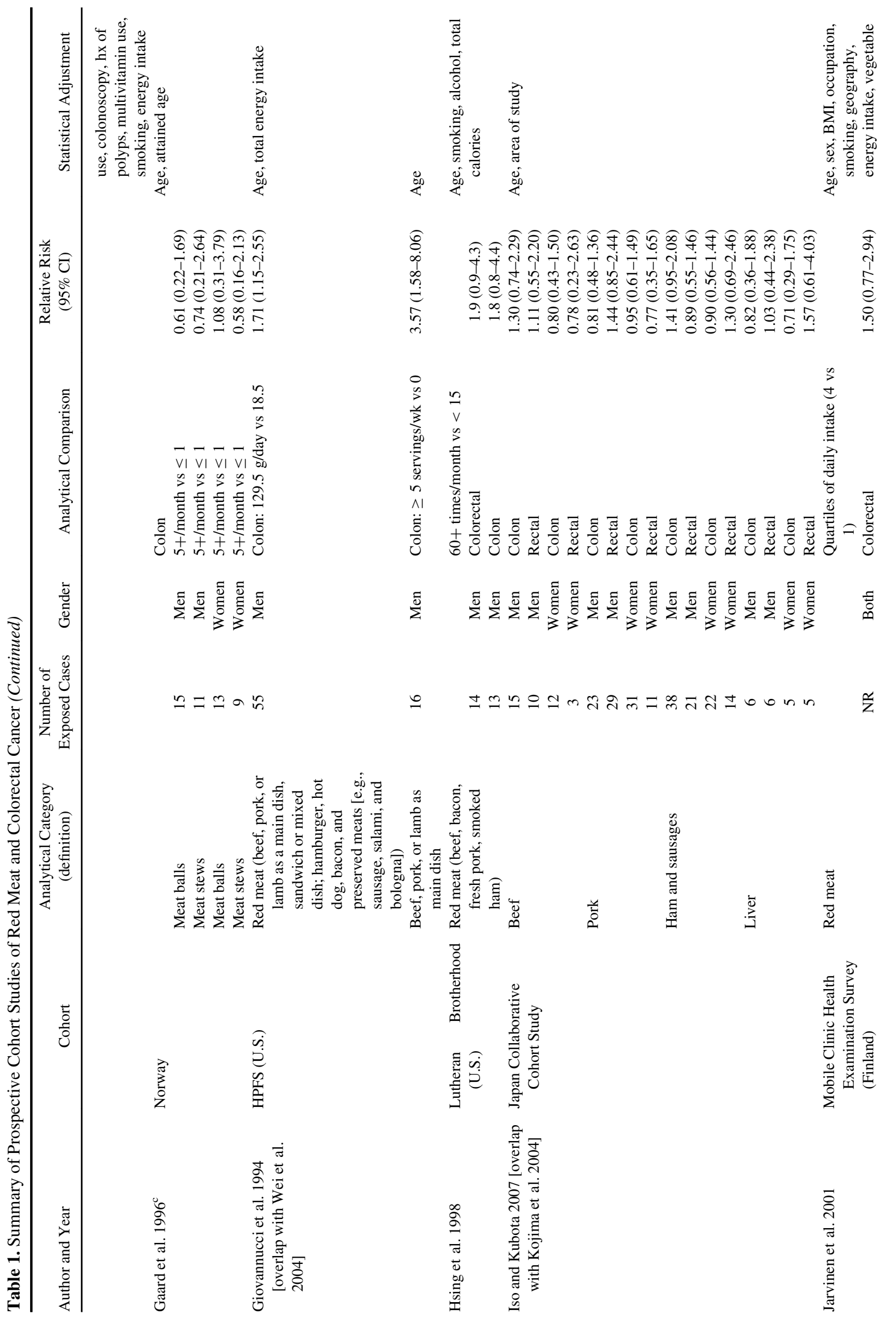




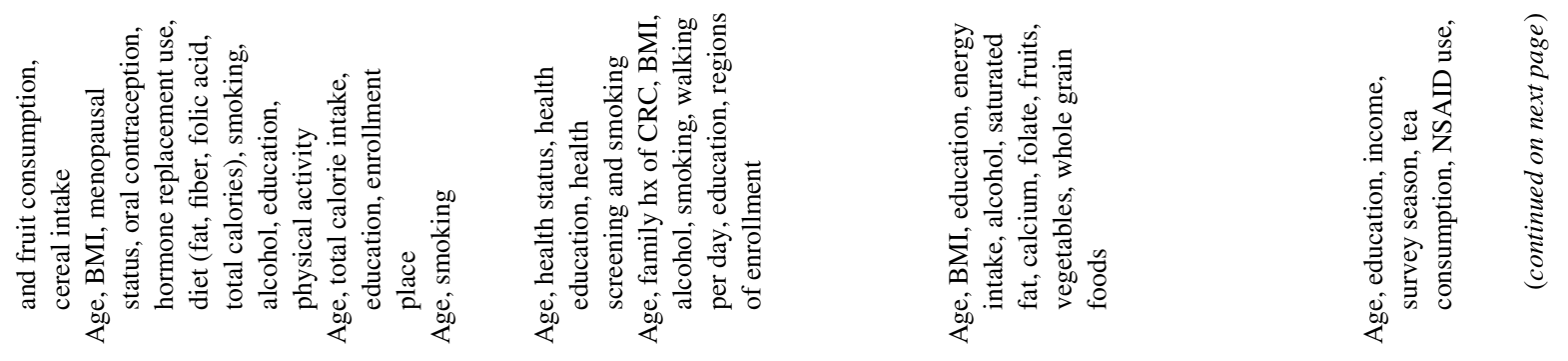

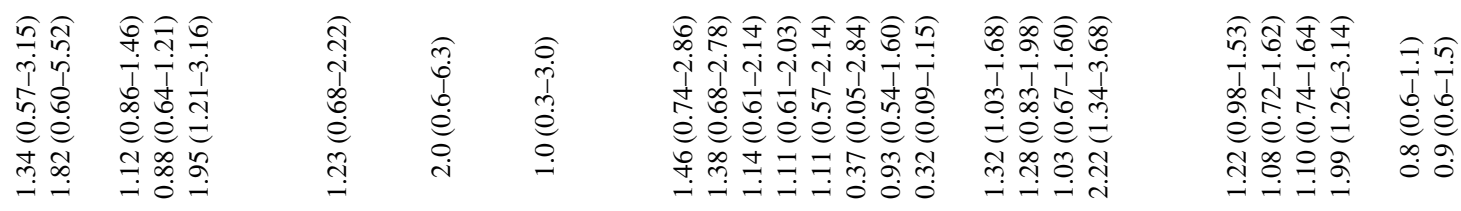

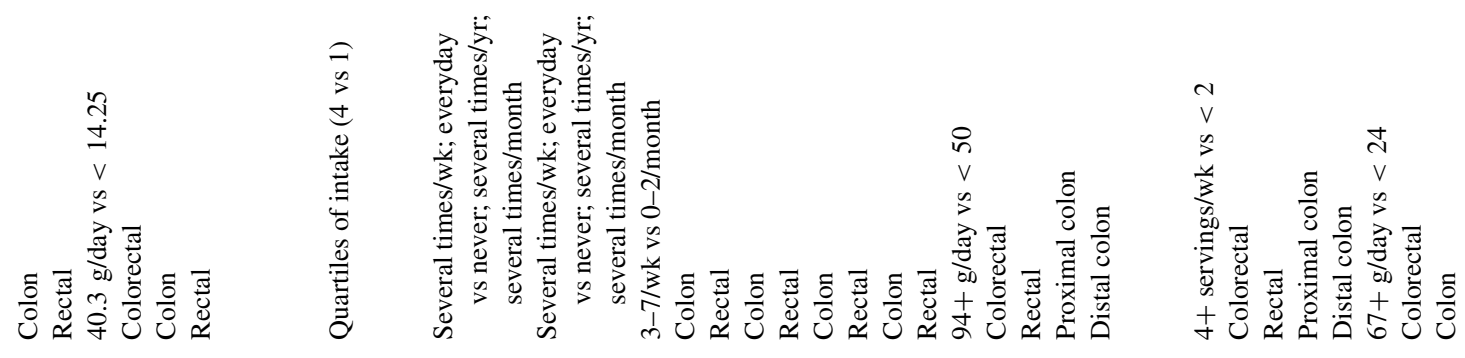

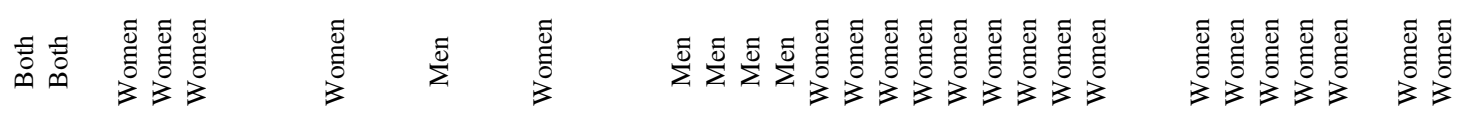


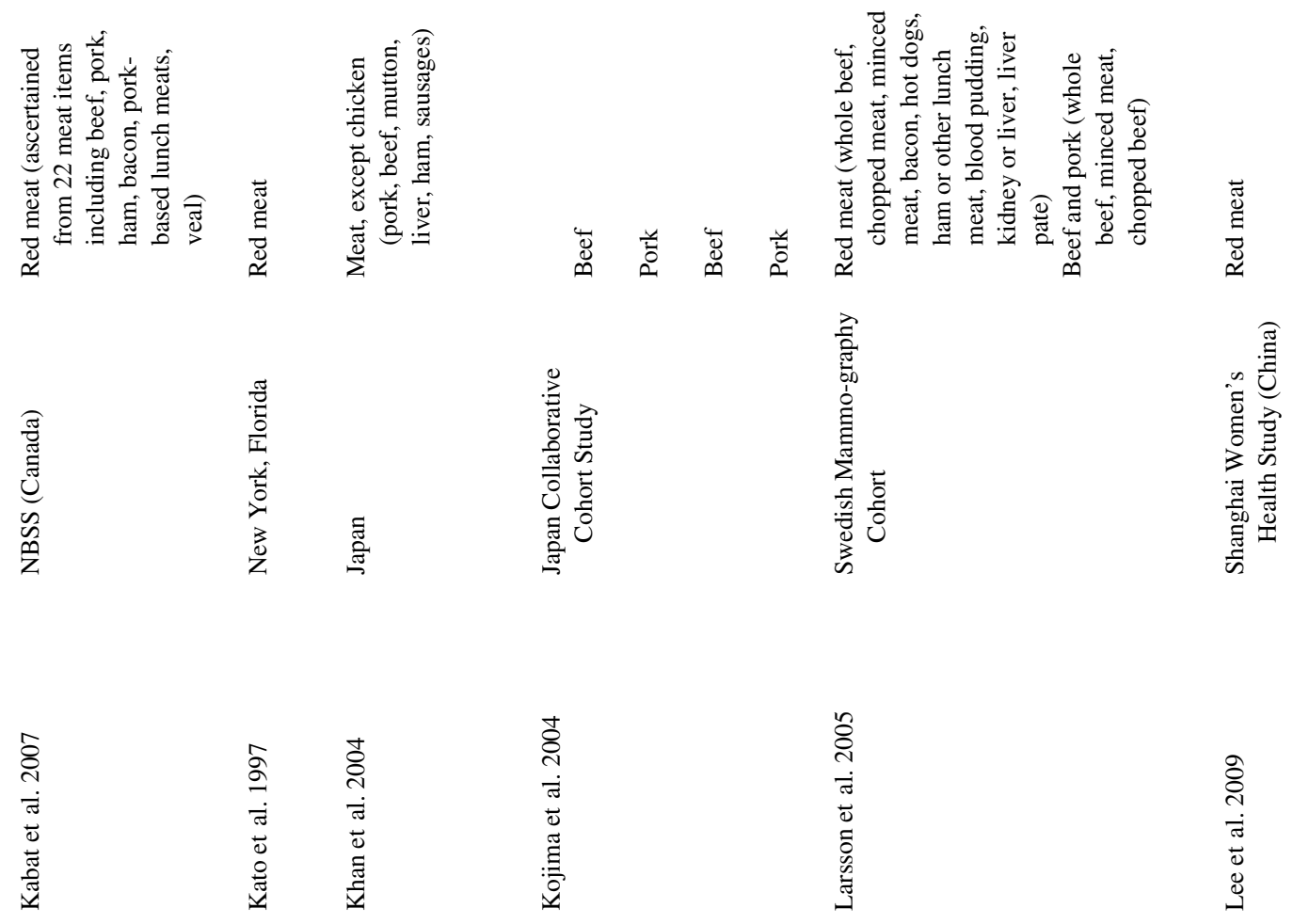






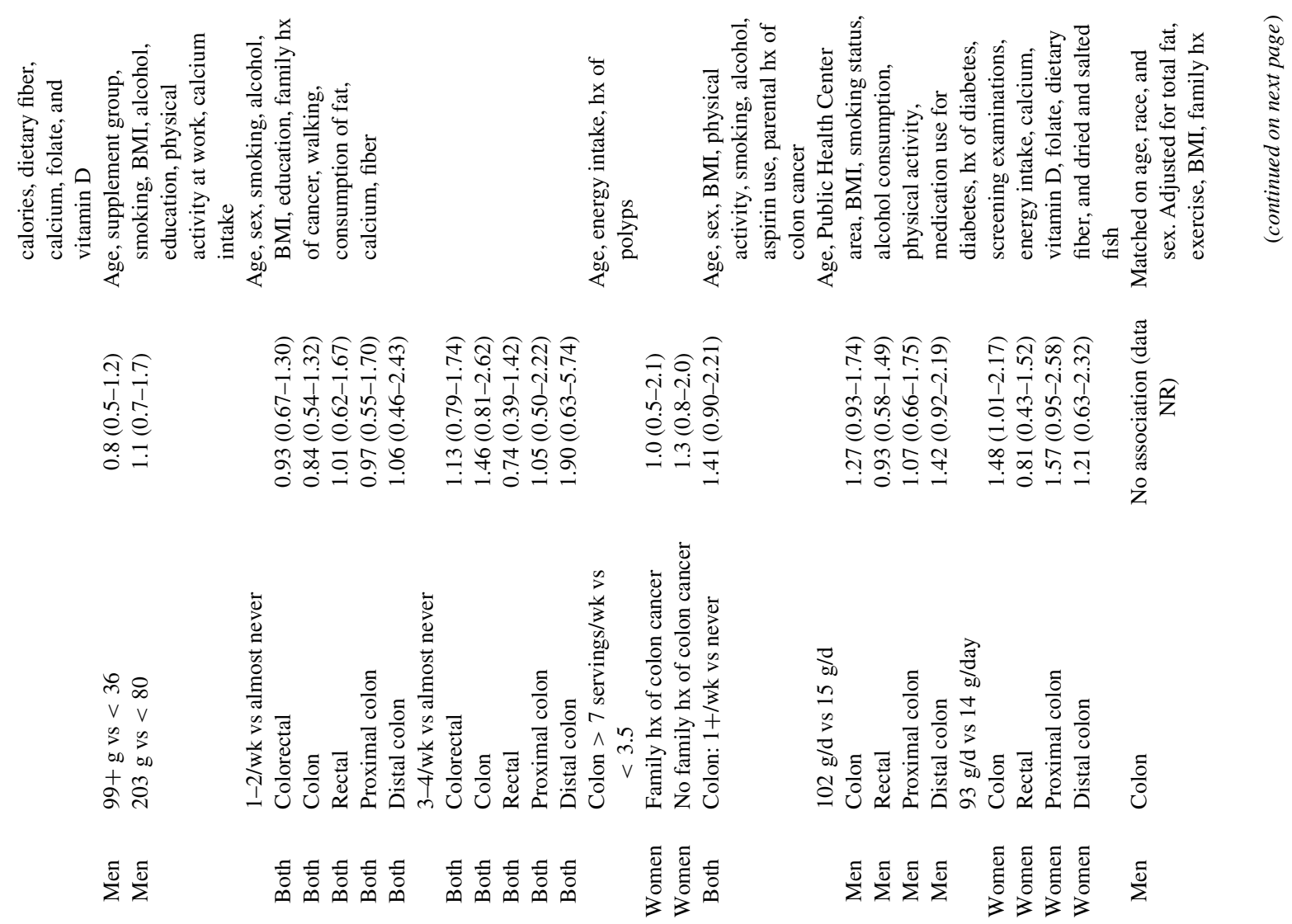

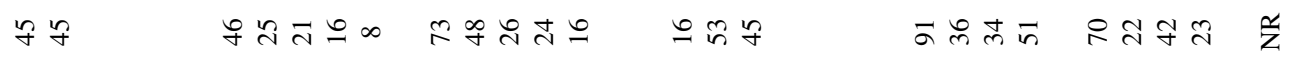
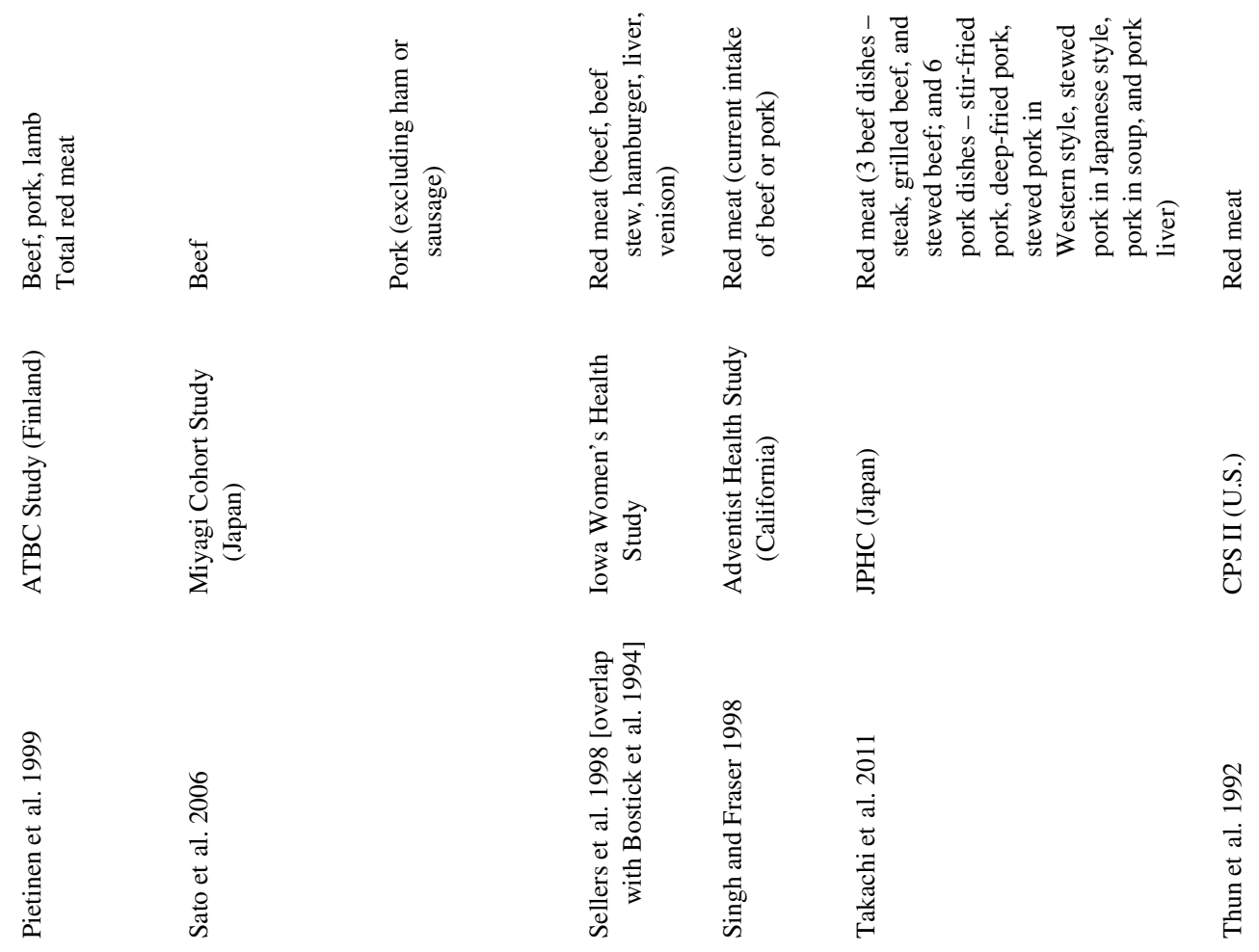


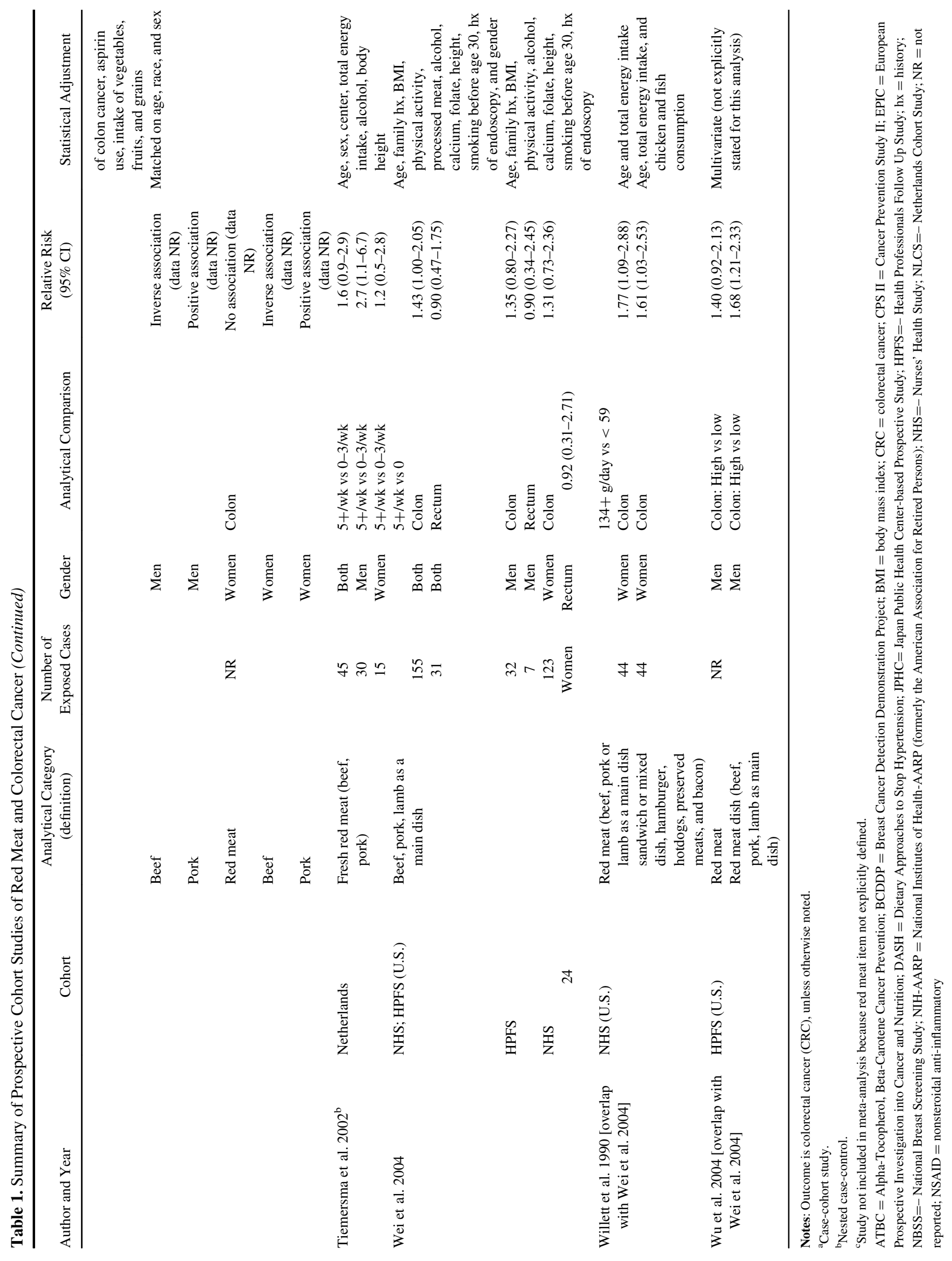


Table 2. Summary of Meta-analysis Results for Red Meat Intake and Colorectal Cancer

\begin{tabular}{|c|c|c|c|c|}
\hline Model (number of studies) & SRRE & $95 \% \mathrm{CI}$ & $\begin{array}{c}P \text {-value for } \\
\text { Heterogeneity and } I^{2}\end{array}$ & Analytical Notes \\
\hline All studies $(n=27)$ & 1.11 & $1.03-1.19$ & $0.014 ; I^{2}=33.60$ & $\begin{array}{l}\text { Includes men and women, colon and rectal } \\
\text { tumor sites }\end{array}$ \\
\hline $\begin{array}{l}\text { All studies, excluding Chen et al. 2003, } \\
\text { Singh and Fraser 1998, and Khan et al. } \\
2004(n=24)\end{array}$ & 1.10 & $1.03-1.18$ & $0.010 ; I^{2}=36.30$ & $\begin{array}{l}\text { Includes men and women, colon and rectal } \\
\text { tumor sites }\end{array}$ \\
\hline $\begin{array}{l}\text { Studies including red meat independent of } \\
\text { processed meat }(n=17)\end{array}$ & 1.05 & $0.98-1.12$ & $0.328 ; I^{2}=8.451$ & $\begin{array}{l}\text { Includes studies that reported a "red meat" } \\
\text { category independent of processed meat }\end{array}$ \\
\hline $\begin{array}{l}\text { Studies including red meat, explicitly } \\
\quad \text { defined }(n=13)\end{array}$ & 1.04 & $0.98-1.14$ & $0.263 ; I^{2}=13.47$ & $\begin{array}{l}\text { Includes studies that explicitly defined "red } \\
\text { meat" }\end{array}$ \\
\hline $\begin{array}{l}\text { Category of red meat intake }[<40 \mathrm{~g} / \text { day }] \\
\quad(n=9)\end{array}$ & 1.02 & $0.98-1.07$ & $0.912 ; I^{2}=0.00$ & $\begin{array}{l}\text { Includes studies that reported median red } \\
\text { meat intake less than } 40 \text { g/day } \\
\text { [Ollberding } 2012 \text { replaces Nothlings } \\
\text { 2009] }\end{array}$ \\
\hline $\begin{array}{l}\text { Category of red meat intake [ } 40-70 \mathrm{~g} / \text { day }] \\
\quad(n=12)\end{array}$ & 1.00 & $0.96-1.04$ & $0.485 ; I^{2}=0.00$ & $\begin{array}{l}\text { Includes studies that reported median red } \\
\text { meat intake between } 40 \text { and } 70 \text { g/day } \\
\text { [Ollberding } 2012 \text { replaces Nothlings } \\
\text { 2009] }\end{array}$ \\
\hline $\begin{array}{l}\text { Category of red meat intake [71-100 g/day] } \\
\quad(n=8)\end{array}$ & 1.03 & $0.89-1.18$ & $0.008 ; I^{2}=59.65$ & $\begin{array}{l}\text { Includes studies that reported median red } \\
\text { meat intake between } 71 \text { and } 100 \mathrm{~g} / \text { day }\end{array}$ \\
\hline $\begin{array}{l}\text { Category of red meat intake [> } 100 / \text { day }] \\
\quad(n=6)\end{array}$ & 1.20 & $1.11-1.29$ & $0.773 ; I^{2}=0.00$ & $\begin{array}{l}\text { Includes studies that reported median red } \\
\text { meat intake greater than } 100 \mathrm{~g} / \text { day }\end{array}$ \\
\hline $\begin{array}{l}\text { Category of red meat intake [0-1 servings/ } \\
\quad \text { week] }(n=4)\end{array}$ & 1.17 & $1.07-1.28$ & $0.575 ; I^{2}=0.00$ & $\begin{array}{l}\text { Includes studies that reported red meat } \\
\text { intake between } 0 \text { and } 1 \text { serving/week [Iso } \\
2007 \text { replaces Kojima 2004] }\end{array}$ \\
\hline $\begin{array}{l}\text { Category of red meat intake [2-4 servings/ } \\
\quad \text { week] }(n=8)\end{array}$ & 1.11 & $1.01-1.22$ & $0.609 ; I^{2}=0.00$ & $\begin{array}{l}\text { Includes studies that reported red meat } \\
\text { intake between } 2 \text { and } 4 \text { servings/week } \\
\text { [Iso } 2007 \text { replaces Kojima 2004] }\end{array}$ \\
\hline $\begin{array}{l}\text { Category of red meat intake }[5-7 \text { servings/ } \\
\quad \text { week] }(n=8)\end{array}$ & 1.22 & $1.07-1.39$ & $0.504 ; I^{2}=0.00$ & $\begin{array}{l}\text { Includes studies that reported red meat } \\
\text { intake between } 5 \text { and } 7 \text { servings/week }\end{array}$ \\
\hline $\begin{array}{l}\text { Category of red meat intake [ }>7 \text { servings/ } \\
\quad \text { week] }(n=5)\end{array}$ & 1.13 & $0.93-1.39$ & $0.215 ; I^{2}=26.75$ & $\begin{array}{l}\text { Includes studies that reported red meat } \\
\text { intake greater than } 7 \text { servings/week }\end{array}$ \\
\hline Adjusted for 3 factors $(n=17)$ & 1.09 & $1.01-1.17$ & $0.005 ; I^{2}=42.171$ & $\begin{array}{l}\text { Includes only studies that reported adjusting } \\
\text { simultaneously for at least } 3 \text { of the } \\
\text { following factors: total energy, BMI, } \\
\text { physical activity, alcohol, family hx of } \\
\text { cancer, education, income (SES) } \\
\text { [Ollberding } 2012 \text { replaces Nothlings 2009] }\end{array}$ \\
\hline $\begin{array}{l}\text { Adjusted for } 3 \text { factors, excluding Singh and } \\
\text { Fraser } 1998(n=16)\end{array}$ & 1.08 & $1.00-1.17$ & $0.005 ; I^{2}=42.65$ & $\begin{array}{l}\text { Includes only studies that reported adjusting } \\
\text { simultaneously for at least } 3 \text { of the } \\
\text { following factors: total energy, BMI, } \\
\text { physical activity, alcohol, family hx of } \\
\text { cancer, education, income (SES) } \\
\text { [Ollberding } 2012 \text { replaces Nothlings 2009] }\end{array}$ \\
\hline $\begin{array}{l}\text { Dose-response: each incremental serving per } \\
\quad \text { week }(n=10)\end{array}$ & 1.02 & $1.00-1.04$ & $0.075 ; I^{2}=26.56$ & $\begin{array}{l}\text { Studies that reported data in a servings per } \\
\text { week metric }\end{array}$ \\
\hline $\begin{array}{l}\text { Dose-response: each } 70 \text { gram increment } \\
\quad(n=13)\end{array}$ & 1.05 & $0.97-1.13$ & $<0.001 ; I^{2}=88.62$ & $\begin{array}{l}\text { Studies that reported data in a grams per day } \\
\text { metric }\end{array}$ \\
\hline Colon $(n=16)$ & 1.11 & $1.04-1.18$ & $0.588 ; I^{2}=0.00$ & $\begin{array}{l}\text { Includes data reported specifically for colon } \\
\text { cancer, men and women included [Iso } \\
2007 \text { replaces Kojima 2004] }\end{array}$ \\
\hline $\begin{array}{l}\text { Colon, excluding Chen et al. } 2003 \text { and Singh } \\
\text { and Fraser } 1998(n=14)\end{array}$ & 1.10 & $1.03-1.17$ & $0.610 ; I^{2}=0.00$ & $\begin{array}{l}\text { Includes data reported specifically for colon } \\
\text { cancer, men and women included [Iso } \\
2007 \text { replaces Kojima 2004] }\end{array}$ \\
\hline Colon, adjusted for 3 factors $(n=12)$ & 1.12 & $1.04-1.20$ & $0.542 ; I^{2}=0.00$ & $\begin{array}{l}\text { Includes only studies that reported adjusting } \\
\text { simultaneously for at least } 3 \text { of the } \\
\text { following factors: total energy, BMI, } \\
\text { physical activity, alcohol, family hx of } \\
\text { cancer, education, income (SES) }\end{array}$ \\
\hline
\end{tabular}




\section{Red Meat and Colorectal Cancer}

Table 2. Summary of Meta-analysis Results for Red Meat Intake and Colorectal Cancer (Continued)

\begin{tabular}{|c|c|c|c|c|}
\hline Model (number of studies) & SRRE & $95 \% \mathrm{CI}$ & $\begin{array}{c}P \text {-value for } \\
\text { Heterogeneity and } I^{2}\end{array}$ & Analytical Notes \\
\hline $\begin{array}{l}\text { Colon, adjusted for } 3 \text { factors, excluding } \\
\text { Singh and Fraser } 1998(n=11)\end{array}$ & 1.11 & $1.04-1.19$ & $0.552 ; I^{2}=0.00$ & $\begin{array}{l}\text { Includes only studies that reported adjusting } \\
\text { simultaneously for at least } 3 \text { of the } \\
\text { following factors: total energy, BMI, } \\
\text { physical activity, alcohol, family hx of } \\
\text { cancer, education, income (SES) }\end{array}$ \\
\hline $\begin{array}{l}\text { Colon, red meat independent of processed } \\
\text { meat }(n=11)\end{array}$ & 1.06 & $0.97-1.16$ & $0.691 ; I^{2}=0.00$ & $\begin{array}{l}\text { Includes studies that reported a "red meat" } \\
\text { category independent of processed meat }\end{array}$ \\
\hline $\operatorname{Rectal}(n=13)$ & 1.17 & $0.99-1.39$ & $0.008 ; I^{2}=51.97$ & $\begin{array}{l}\text { Includes data reported specifically for rectal } \\
\text { cancer, men and women included [Iso } \\
2007 \text { replaces Kojima 2004] }\end{array}$ \\
\hline Rectal, adjusted for 3 factors $(n=11)$ & 1.09 & $0.90-1.32$ & $0.003 ; I^{2}=58.35$ & $\begin{array}{l}\text { Includes only studies that reported adjusting } \\
\text { simultaneously for at least } 3 \text { of the } \\
\text { following factors: total energy, BMI, } \\
\text { physical activity, alcohol, family hx of } \\
\text { cancer, education, income (SES) }\end{array}$ \\
\hline $\begin{array}{l}\text { Rectal, red meat independent of processed } \\
\text { meat }(n=10)\end{array}$ & 1.03 & $0.88-1.21$ & $0.241 ; I^{2}=20.04$ & $\begin{array}{l}\text { Includes studies that reported a "red meat" } \\
\text { category independent of processed meat }\end{array}$ \\
\hline Studies published < $2000(n=8)$ & 1.30 & $1.06-1.59$ & $0.230 ; I^{2}=24.95$ & $\begin{array}{l}\text { Includes only studies published before year } \\
2000 \text { (Willett } 1990 \text { and Giovannucci } \\
1994 \text { replaces Wei 2004) }\end{array}$ \\
\hline $\begin{array}{l}\text { Studies published }<2000 \text {, excluding Singh } \\
\text { and Fraser } 1998(n=7)\end{array}$ & 1.28 & $1.01-1.62$ & $0.164 ; I^{2}=34.59$ & $\begin{array}{l}\text { Includes only studies published before year } \\
2000 \text { (Willett } 1990 \text { and Giovannucci } \\
1994 \text { replaces Wei 2004) }\end{array}$ \\
\hline Studies published 2001-2004 $(n=9)$ & 1.17 & $1.01-1.37$ & $0.212 ; I^{2}=19.84$ & $\begin{array}{l}\text { Includes studies published between } 2001 \text { and } \\
2004\end{array}$ \\
\hline $\begin{array}{l}\text { Studies published 2001-2004, excluding } \\
\text { Chen et al. } 2003 \text { and Khan et al. } 2004 \\
(n=7)\end{array}$ & 1.15 & $0.97-1.37$ & $0.145 ; I^{2}=27.69$ & $\begin{array}{l}\text { Includes studies published between } 2001 \text { and } \\
2004\end{array}$ \\
\hline Studies published 2005-2008 $(n=10)$ & 1.11 & $1.01-1.21$ & $0.013 ; I^{2}=45.93$ & $\begin{array}{l}\text { Includes studies published between } 2005 \text { and } \\
2004\end{array}$ \\
\hline Studies published $>2009(n=3)$ & 1.03 & $0.87-1.22$ & $0.150 ; I^{2}=36.46$ & $\begin{array}{l}\text { Includes studies published after } 2009 \\
\text { (Ollberding } 2012 \text { replaces Nothlings } \\
\text { 2009) }\end{array}$ \\
\hline $\begin{array}{l}\text { Studies published in the past } 5 \text { years } \\
\text { [2007-2012] }(n=7)\end{array}$ & 1.09 & $0.98-1.21$ & $0.005 ; I^{2}=54.19$ & $\begin{array}{l}\text { Includes studies published in the past } 5 \text { years } \\
\text { (Ollberding } 2012 \text { replaces Nothlings } \\
\text { 2009) }\end{array}$ \\
\hline North America (U.S. \& Canada) $(n=12)$ & 1.18 & $1.05-1.32$ & $0.012 ; I^{2}=48.32$ & $\begin{array}{l}\text { Studies conducted among U.S. or Canadian } \\
\text { populations [Ollberding } 2012 \text { replaces } \\
\text { Nothlings 2009] }\end{array}$ \\
\hline $\begin{array}{l}\text { North America (U.S. \& Canada), excluding } \\
\quad \text { Singh and Fraser } 1998(n=11)\end{array}$ & 1.17 & $1.04-1.31$ & $0.010 ; I^{2}=50.02$ & $\begin{array}{l}\text { Studies conducted among U.S. or Canadian } \\
\text { populations [Ollberding } 2012 \text { replaces } \\
\text { Nothlings 2009] }\end{array}$ \\
\hline All other countries $(n=15)$ & 1.07 & $0.98-1.16$ & $0.187 ; I^{2}=18.40$ & $\begin{array}{l}\text { Studies conducted in Europe, Japan, China, } \\
\text { Australia [Iso } 2007 \text { replaces Kojima } \\
\text { 2004] }\end{array}$ \\
\hline $\begin{array}{l}\text { All other countries, excluding Chen et al. } \\
2003 \text { and Khan et al. } 2004(n=13)\end{array}$ & 1.06 & $0.97-1.15$ & $0.163 ; I^{2}=21.17$ & $\begin{array}{l}\text { Studies conducted in Europe, Japan, China, } \\
\text { Australia [Iso } 2007 \text { replaces Kojima } \\
\text { 2004] }\end{array}$ \\
\hline Europe $(n=6)$ & 1.09 & $0.94-1.27$ & $0.132 ; I^{2}=34.47$ & $\begin{array}{l}\text { Studies conducted in Finland, the } \\
\text { Netherlands, Sweden, and other countries } \\
\text { (EPIC) }\end{array}$ \\
\hline Asia $(n=8)$ & 1.03 & $0.94-1.13$ & $0.557 ; I^{2}=0.00$ & $\begin{array}{l}\text { Studies conducted in Japan and China [Iso } \\
2007 \text { replaces Kojima 2004] }\end{array}$ \\
\hline $\begin{array}{l}\text { Asia, excluding Chen et al. } 2003 \text { and Khan } \\
\text { et al. } 2004(n=6)\end{array}$ & 1.01 & $0.92-1.12$ & $0.558 ; I^{2}=0.00$ & $\begin{array}{l}\text { Studies conducted in Japan and China [Iso } \\
2007 \text { replaces Kojima 2004] }\end{array}$ \\
\hline $\operatorname{Men}(n=10)$ & 1.16 & $1.02-1.32$ & $0.439 ; I^{2}=0.648$ & $\begin{array}{l}\text { Studies that reported data specifically for } \\
\text { men [Iso } 2007 \text { replaces Kojima 2004] }\end{array}$ \\
\hline Men, excluding Khan $2004(n=9)$ & 1.15 & $1.01-1.32$ & $0.423 ; I^{2}=2.21$ & $\begin{array}{l}\text { Studies that reported data specifically for } \\
\text { men [Iso } 2007 \text { replaces Kojima 2004] }\end{array}$ \\
\hline
\end{tabular}


Table 2. Summary of Meta-analysis Results for Red Meat Intake and Colorectal Cancer (Continued)

\begin{tabular}{|c|c|c|c|c|}
\hline Model (number of studies) & SRRE & $95 \% \mathrm{CI}$ & $\begin{array}{c}P \text {-value for } \\
\text { Heterogeneity and } I^{2}\end{array}$ & Analytical Notes \\
\hline Men, adjusted for 3 factors $(n=6)$ & 1.15 & $0.99-1.33$ & $0.714 ; I^{2}=0.00$ & $\begin{array}{l}\text { Includes only studies that reported adjusting } \\
\text { simultaneously for at least } 3 \text { of the } \\
\text { following factors: total energy, BMI, } \\
\text { physical activity, alcohol, family hx of } \\
\text { cancer, education, income (SES) }\end{array}$ \\
\hline $\begin{array}{l}\text { Men, red meat independent of processed } \\
\text { meat }(n=6)\end{array}$ & 1.11 & $0.94-1.30$ & $0.353 ; I^{2}=9.90$ & $\begin{array}{l}\text { Includes studies that reported a "red meat" } \\
\text { category independent of processed meat }\end{array}$ \\
\hline Men, Colon $(n=5)$ & 1.18 & $0.99-1.39$ & $0.676 ; I^{2}=0.00$ & $\begin{array}{l}\text { Studies that reported data for colon cancer } \\
\text { among men [Iso } 2007 \text { replaces Kojima } \\
\text { 2004] }\end{array}$ \\
\hline Men, $\operatorname{Rectal}(n=3)$ & 1.09 & $0.83-1.45$ & $0.584 ; I^{2}=0.00$ & $\begin{array}{l}\text { Studies that reported data for rectal cancer } \\
\text { among men [Iso } 2007 \text { replaces Kojima } \\
\text { 2004] }\end{array}$ \\
\hline $\begin{array}{l}\text { Men, category of red meat intake } \\
\quad[<40 \mathrm{~g} / \text { day }](n=3)\end{array}$ & 1.11 & $0.94-1.30$ & $0.899 ; I^{2}=0.00$ & $\begin{array}{l}\text { Includes studies that reported median red } \\
\text { meat intake less than } 40 \mathrm{~g} / \text { day }\end{array}$ \\
\hline $\begin{array}{l}\text { Men, category of red meat intake } \\
\quad[40-70 \mathrm{~g} / \mathrm{day}](n=4)\end{array}$ & 1.05 & $0.92-1.19$ & $0.348 ; I^{2}=10.52$ & $\begin{array}{l}\text { Includes studies that reported median red } \\
\text { meat intake between } 40 \text { and } 70 \mathrm{~g} / \text { day }\end{array}$ \\
\hline $\begin{array}{l}\text { Men, category of red meat intake } \\
\quad[71-100 \mathrm{~g} / \mathrm{day}](n=2)\end{array}$ & 1.02 & $0.68-1.54$ & $0.111 ; I^{2}=60.55$ & $\begin{array}{l}\text { Includes studies that reported median red } \\
\text { meat intake between } 71 \text { and } 100 \mathrm{~g} / \text { day }\end{array}$ \\
\hline $\begin{array}{l}\text { Men, category of red meat intake } \\
\quad[>100 \mathrm{~g} / \text { day }](n=3)\end{array}$ & 1.14 & $0.98-1.34$ & $0.819 ; I^{2}=0.00$ & $\begin{array}{l}\text { Includes studies that reported median red } \\
\text { meat intake greater than } 100 \mathrm{~g} / \text { day }\end{array}$ \\
\hline $\begin{array}{l}\text { Men, category of red meat intake } \\
{[0-1 \text { servings/week }](n=2)}\end{array}$ & 1.31 & $1.12-1.54$ & $0.988 ; I^{2}=0.00$ & $\begin{array}{l}\text { Includes studies that reported red meat } \\
\text { intake between } 0 \text { and } 1 \text { serving/week [Iso } \\
2007 \text { replaces Kojima 2004] }\end{array}$ \\
\hline $\begin{array}{l}\text { Men, category of red meat intake } \\
{[2-4 \text { servings/week] }(n=4)}\end{array}$ & 1.27 & $1.04-1.56$ & $0.515 ; I^{2}=0.00$ & $\begin{array}{l}\text { Includes studies that reported red meat } \\
\text { intake between } 2 \text { and } 4 \text { servings/week } \\
\text { [Iso } 2007 \text { replaces Kojima 2004] }\end{array}$ \\
\hline $\begin{array}{l}\text { Men, category of red meat intake } \\
{[5-7 \text { servings/week }](n=4)}\end{array}$ & 1.29 & $0.96-1.74$ & $0.289 ; I^{2}=19.76$ & $\begin{array}{l}\text { Includes studies that reported red meat } \\
\text { intake between } 5 \text { and } 7 \text { servings/week }\end{array}$ \\
\hline $\begin{array}{l}\text { Men, category of red meat intake } \\
\quad[>7 \text { servings/week] }(n=2)\end{array}$ & 1.38 & $0.97-1.96$ & $0.607 ; I^{2}=0.00$ & $\begin{array}{l}\text { Includes studies that reported red meat } \\
\text { intake greater than } 7 \text { servings/week }\end{array}$ \\
\hline $\begin{array}{l}\text { Dose-response: each in cremental serving } \\
\text { per week }(n=5)\end{array}$ & 1.04 & $1.01-1.06$ & 0.511 & $\begin{array}{l}\text { Studies that reported data in a servings per } \\
\text { week metric }\end{array}$ \\
\hline $\begin{array}{l}\text { Dose-response: each } 70 \text { gram increment } \\
\quad(n=3)\end{array}$ & 1.01 & $0.77-1.33$ & 0.021 & $\begin{array}{l}\text { Studies that reported data in a grams per day } \\
\text { metric }\end{array}$ \\
\hline Women $(n=14)$ & 1.03 & $0.91-1.17$ & $0.124 ; I^{2}=28.09$ & $\begin{array}{l}\text { Studies that reported data specifically for } \\
\text { women [Iso } 2007 \text { replaces Kojima 2004] }\end{array}$ \\
\hline Women, excluding Khan $2004(n=13)$ & 1.03 & $0.91-1.18$ & $0.094 ; I^{2}=32.07$ & $\begin{array}{l}\text { Studies that reported data specifically for } \\
\text { women [Iso } 2007 \text { replaces Kojima 2004] }\end{array}$ \\
\hline Women, adjusted for 3 factors $(n=10)$ & 1.01 & $0.85-1.19$ & $0.016 ; I^{2}=49.49$ & $\begin{array}{l}\text { Includes only studies that reported adjusting } \\
\text { simultaneously for at least } 3 \text { of the } \\
\text { following factors: total energy, BMI, } \\
\text { physical activity, alcohol, family hx of } \\
\text { cancer, education, income (SES) }\end{array}$ \\
\hline $\begin{array}{l}\text { Women, red meat independent of processed } \\
\text { meat }(n=9)\end{array}$ & 1.06 & $0.92-1.22$ & $0.308 ; I^{2}=13.60$ & $\begin{array}{l}\text { Includes studies that reported a "red meat" } \\
\text { category independent of processed meat }\end{array}$ \\
\hline Women, Colon $(n=8)$ & 0.99 & $0.85-1.15$ & $0.400 ; I^{2}=3.91$ & $\begin{array}{l}\text { Studies that reported data for colon cancer } \\
\text { among women [Iso } 2007 \text { replaces Kojima } \\
\text { 2004] }\end{array}$ \\
\hline Women, Rectal $(n=6)$ & 1.07 & $0.76-1.51$ & $0.064 ; I^{2}=52.07$ & $\begin{array}{l}\text { Studies that reported data for rectal cancer } \\
\text { among women [Iso } 2007 \text { replaces Kojima } \\
\text { 2004] }\end{array}$ \\
\hline $\begin{array}{l}\text { Women, category of red meat intake } \\
\qquad[<40 \mathrm{~g} / \text { day }](n=6)\end{array}$ & 1.00 & $0.91-1.10$ & $0.443 ; I^{2}=0.00$ & $\begin{array}{l}\text { Includes studies that reported median red } \\
\text { meat intake less than } 40 \mathrm{~g} / \text { day }\end{array}$ \\
\hline $\begin{array}{l}\text { Women, category of red meat intake } \\
{[40-70 \mathrm{~g} / \text { day }](n=7)}\end{array}$ & 0.97 & $0.89-1.07$ & $0.371 ; I^{2}=7.60$ & $\begin{array}{l}\text { Includes studies that reported median red } \\
\text { meat intake between } 40 \text { and } 70 \mathrm{~g} / \text { day }\end{array}$ \\
\hline $\begin{array}{l}\text { Women, category of red meat intake } \\
{[71-100 \mathrm{~g} / \text { day }](n=3)}\end{array}$ & 1.04 & $0.80-1.36$ & $0.067 ; I^{2}=58.19$ & $\begin{array}{l}\text { Includes studies that reported median red } \\
\text { meat intake between } 71 \text { and } 100 \mathrm{~g} / \text { day }\end{array}$ \\
\hline
\end{tabular}


Table 2. Summary of Meta-analysis Results for Red Meat Intake and Colorectal Cancer (Continued)

\begin{tabular}{|c|c|c|c|c|}
\hline Model (number of studies) & SRRE & $95 \%$ CI & $\begin{array}{c}P \text {-value for } \\
\text { Heterogeneity and } I^{2}\end{array}$ & Analytical Notes \\
\hline $\begin{array}{l}\text { Women, category of red meat intake } \\
\qquad>100 \mathrm{~g} / \text { day }](n=3)\end{array}$ & 1.14 & $0.95-1.37$ & $0.0 .301 ; I^{2}=16.62$ & $\begin{array}{l}\text { Includes studies that reported median red } \\
\text { meat intake greater than } 100 \mathrm{~g} / \text { day }\end{array}$ \\
\hline $\begin{array}{l}\text { Women, category of red meat intake } \\
\text { [0-1 servings/week] }(n=2)\end{array}$ & 0.92 & $0.75-1.12$ & $0.692 ; I^{2}=0.00$ & $\begin{array}{l}\text { Includes studies that reported red meat } \\
\text { intake between } 0 \text { and } 1 \text { serving/week [Iso } \\
2007 \text { replaces Kojima 2004] }\end{array}$ \\
\hline $\begin{array}{l}\text { Women, category of red meat intake } \\
{[2-4 \text { servings/week }](n=5)}\end{array}$ & 1.02 & $0.91-1.15$ & $0.898 ; I^{2}=0.00$ & $\begin{array}{l}\text { Includes studies that reported red meat } \\
\text { intake between } 2 \text { and } 4 \text { servings/week } \\
\text { [Iso } 2007 \text { replaces Kojima 2004] }\end{array}$ \\
\hline $\begin{array}{l}\text { Women, category of red meat intake } \\
{[5-7 \text { servings/week }](n=5)}\end{array}$ & 1.14 & $0.96-1.34$ & $0.708 ; I^{2}=0.00$ & $\begin{array}{l}\text { Includes studies that reported red meat } \\
\text { intake between } 5 \text { and } 7 \text { servings/week }\end{array}$ \\
\hline $\begin{array}{l}\text { Women, category of red meat intake } \\
\text { [> } 7 \text { servings/week }](n=2)\end{array}$ & 0.93 & $0.72-1.20$ & $0.349 ; I^{2}=8.83$ & $\begin{array}{l}\text { Includes studies that reported red meat } \\
\text { intake greater than } 7 \text { servings/week }\end{array}$ \\
\hline $\begin{array}{l}\text { Dose-response: each incremental serving per } \\
\quad \text { week }(n=6)\end{array}$ & 1.00 & $0.97-1.03$ & $0.135 ; I^{2}=16.89$ & $\begin{array}{l}\text { Studies that reported data in a servings per } \\
\text { week metric }\end{array}$ \\
\hline $\begin{array}{l}\text { Dose-response: each } 70 \text { gram increment } \\
\quad(n=6)\end{array}$ & 1.00 & $0.82-1.21$ & $<0.001 ; I^{2}=86.25$ & $\begin{array}{l}\text { Studies that reported data in a grams per day } \\
\text { metric }\end{array}$ \\
\hline
\end{tabular}

separately in numerous studies as well. Of the 48 individual point estimates (for the highest intake level in each study) reflecting data from 27 individual studies, only eight associations were statistically significant in the positive direction. Over one-third ( $n=18$ ) of the individual RRs were 1.0 or lower, and almost half $(n=23,48 \%)$ of the RRs were less than 1.05 .

The meta-analysis results are summarized in Table 2 (based on high vs low intake unless otherwise specified by intake levels).

\section{High vs Low Meta-Analysis Results}

The SRRE for all 27 prospective studies of high vs low red meat intake and CRC was 1.11 (95\% CI: 1.03-1.19); however, as expected, statistically significant heterogeneity was present ( $\mathrm{p}-\mathrm{H}=0.014$ ). When 3 studies that were less specific in their definitions of red meat/exposure categories were removed, the summary effect was largely unchanged, because these studies provided little relative weight $(\mathrm{SRRE}=1.10,95 \%$ CI: 1.03-1.18) (Table 2, Fig. 1). However, in the model that

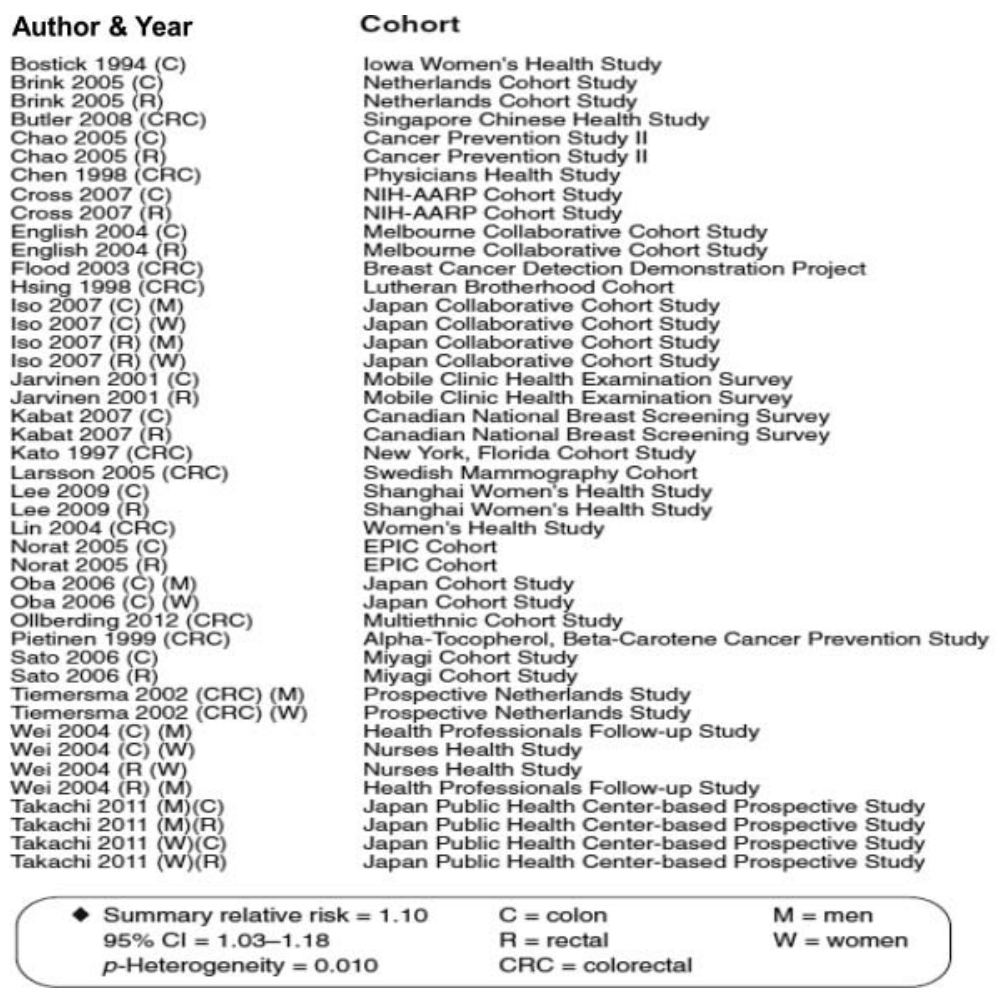

Fig. 1. Meta-analysis of prospective studies of red meat consumption and colorectal cancer.

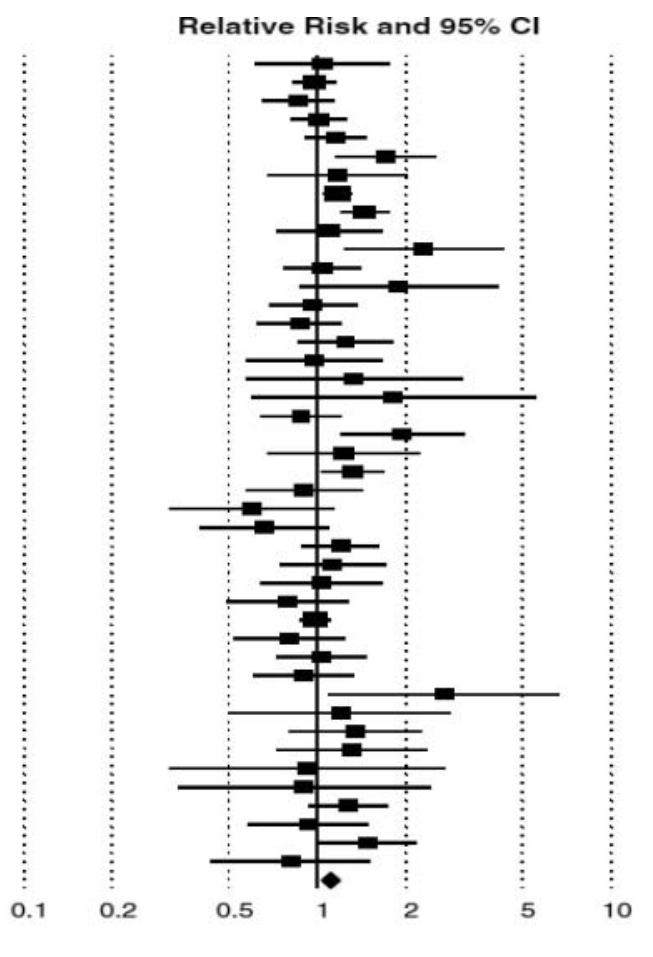


included studies of fresh red meat $(n=17$ studies independent of processed meat items), the SRRE dropped to 1.05 and was no longer statistically significant (95\% CI: 0.98-1.12), and the model was less heterogeneous ( $\mathrm{p}-\mathrm{H}=0.328)$. Of 13 of these studies that explicitly defined red meat, the SRRE was 1.04 (95\% CI: $0.98-1.14, \mathrm{p}-\mathrm{H}=0.263$ ). When restricting the analysis to studies that adjusted simultaneously for at least 3 factors (out of: total energy, body mass index [BMI], physical activity, alcohol, family history of cancer, education, income [socioeconomic status]), the summary association was attenuated slightly $(\mathrm{SRRE}=1.08,95 \% \mathrm{CI}: 1.00-1.17)$. Stronger (and more heterogeneous) associations were observed in meta-analyses of studies conducted in North America (SRRE $=1.18,95 \%$ CI: 1.05-1.32), compared with studies published in other countries $(\mathrm{SRRE}=1.07,95 \%$ CI: 0.98-1.16). The model of studies conducted in China and Japan had the weakest SRRE (1.01, 95\% CI: 0.92-1.12; Chen et al. [22] and Khan et al. [35] excluded). Interestingly, the summary associations were modified by publication date, with the SRREs becoming monotonically weaker over time (Table 2). For example, meta-analysis of 8 studies published prior to the year 2000 resulted in an SRRE of 1.30 (95\% CI: 1.06-1.59), while the SRRE for the studies published in the past 5 years was 1.09 (95\% CI: 0.98-1.21).

Summary associations were stronger and more heterogeneous for rectal cancer $(\mathrm{SRRE}=1.17,95 \% \mathrm{CI}$ : 0.99-1.39, p-H $=0.008)$ compared with colon cancer $(\mathrm{SRRE}=1.11,95 \% \mathrm{CI}$ : $1.04-1.18$, p-H $=0.588$ ). However, the summary effect for rectal cancer attenuated considerably after including studies that adjusted for more relevant factors (SRRE $=1.09$, 95\% CI: $0.90-1.32$; based on studies that simultaneously adjusted for total energy, BMI, physical activity, alcohol, family history of cancer, education, income/socioeconomic status). When the analyses were restricted to studies of fresh red meat, independent of processed meat items, summary associations for both colon cancer (SRRE $=1.06,95 \% \mathrm{CI}: 0.97-1.16$ ) and rectal cancer $(\mathrm{SRRE}=1.03,95 \% \mathrm{CI}: 0.88-1.21)$ became closer to the null value.

We observed modification of summary associations by gender, with stronger (but overall weak to modest) SRREs among men, and associations close to the null value for women. The SRRE for the 10 studies that reported data specifically for men was 1.16 (95\% CI: 1.02-1.32), while the summary association for women was 1.03 (95\% CI: 0.91-1.17). Inconsistent patterns of associations between sexes were observed. Among men, associations were slightly weaker in analyses of red meat (independent of processed meat items) and rectal cancer, but slightly stronger in analyses of colon cancer. In contrast, among women, associations were slightly stronger in analyses of red meat (independent of processed meat items) and rectal cancer, but weaker in analyses of colon cancer. For both men and women, summary effects were slightly weaker for the meta-analyses of studies that adjusted for more relevant potential confounding factors.

\section{Intake Assessment}

Dose-response effects were evaluated using different methods. First, we plotted all point estimates for all intake categories across all studies to generate a visual illustration of all possible data points. This was done for both metrics-grams per day (Fig. 2) and servings per week (Fig. 3). Based on visual examination, no clear pattern of dose-response is apparent. While the absolute number of point estimates above the null value is greater than below, particularly for the servingsper-week metric, no consistent pattern is evident (i.e., increasing point estimates above the null value as intake levels increase). To supplement this visual assessment, we conducted categorical dose-response regression meta-analyses and metaanalyses of categorical intake groupings. The SRRE for each $70 \mathrm{~g}$ increment of red meat was 1.05 (95\% CI: 0.97-1.13), and the SRRE for each incremental serving per week was 1.02 (95\% CI: 1.00-1.04) among men and women combined (both models). No associations for each incremental serving per week (SRRE $=1.00,95 \%$ CI: 0.97-1.03) or for each $70 \mathrm{~g}$ increment of red meat (SRRE $=1.00,95 \% \mathrm{CI}: 0.82-1.21)$ and CRC were observed in the dose-response analyses among women. For men, the SRRE for each incremental serving of red meat per week was 1.04 (95\% CI: 1.01-1.06), and 1.01 (95\% CI: 0.77-1.33) for each $70 \mathrm{~g}$ increment of red meat. No clear pattern of dose-response was observed in our meta-analyses of categorical intake groupings (groups created to approximate common categories across studies) (Table 2; Fig. 4). In doseresponse categories up to $100 \mathrm{~g} /$ day, summary associations ranged between 1.00 and 1.03 and were not statistically significant. However, the SRRE for intake levels above $100 \mathrm{~g} /$ day was 1.20 (95\% CI: 1.11-1.29). There was greater data inflection in the servings-per-week intake groupings, because the summary association for $0-1$ serving of red meat per week was 1.17 , then dropped to 1.11 (2-4 servings), then rose to 1.22 (57 servings), then dropped to 1.13 in the highest category of intake ( $>7$ servings per week). The summary associations for the lowest 3 servings-per-week categories were statistically significant, but the SRRE for the highest level of intake was not statistically significant (Table 2; Fig. 4). The statistically significant findings at the lower intake levels are not substantiated by the findings in the grams-per-day analyses, and there is no apparent reason why patterns of associations would be stronger for servings per week than grams per day. However, in general, studies that reported results for grams-per-day metrics were more analytically specific than studies that reported data in a servings-per-week metric (See Table 1, 3-7/wk vs 02/month in Kojima et al. 2004 [36], for example). Similarly, in our cumulative meta-analysis of increasing grams per day and servings per week, no clear pattern of association was apparent (Fig. 5 and 6). For a dose-response effect to be present, the summary effect would slowly shift to the right (positive direction) as intake increased. Associations appeared to become slightly stronger at the $90 \mathrm{~g} /$ day level; however, this finding 


\section{Red Meat and Colorectal Cancer}

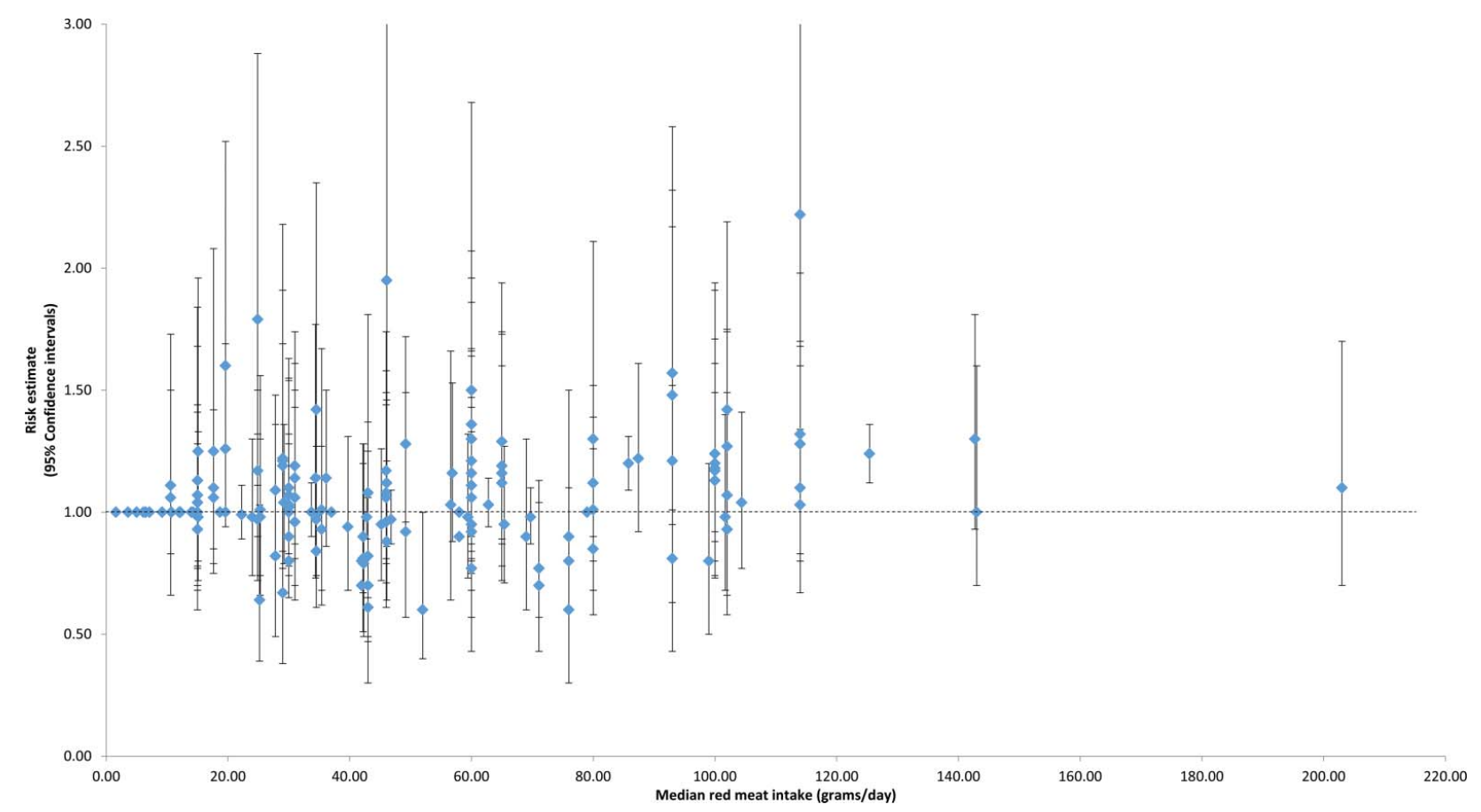

Fig. 2. Individual study relative risks for colorectal cancer across all red meat intake strata (grams/day) in the prospective cohort studies.

was not substantiated by the servings-per-week cumulative meta-analysis, for which the magnitude of associations at low levels of intake were greater than or similar to the magnitude of associations at high intake levels.

\section{Publication Bias}

A visual examination of the funnel plot of prospective studies of red meat and CRC suggested slight publication bias; however, Egger's regression test was not significant (data not

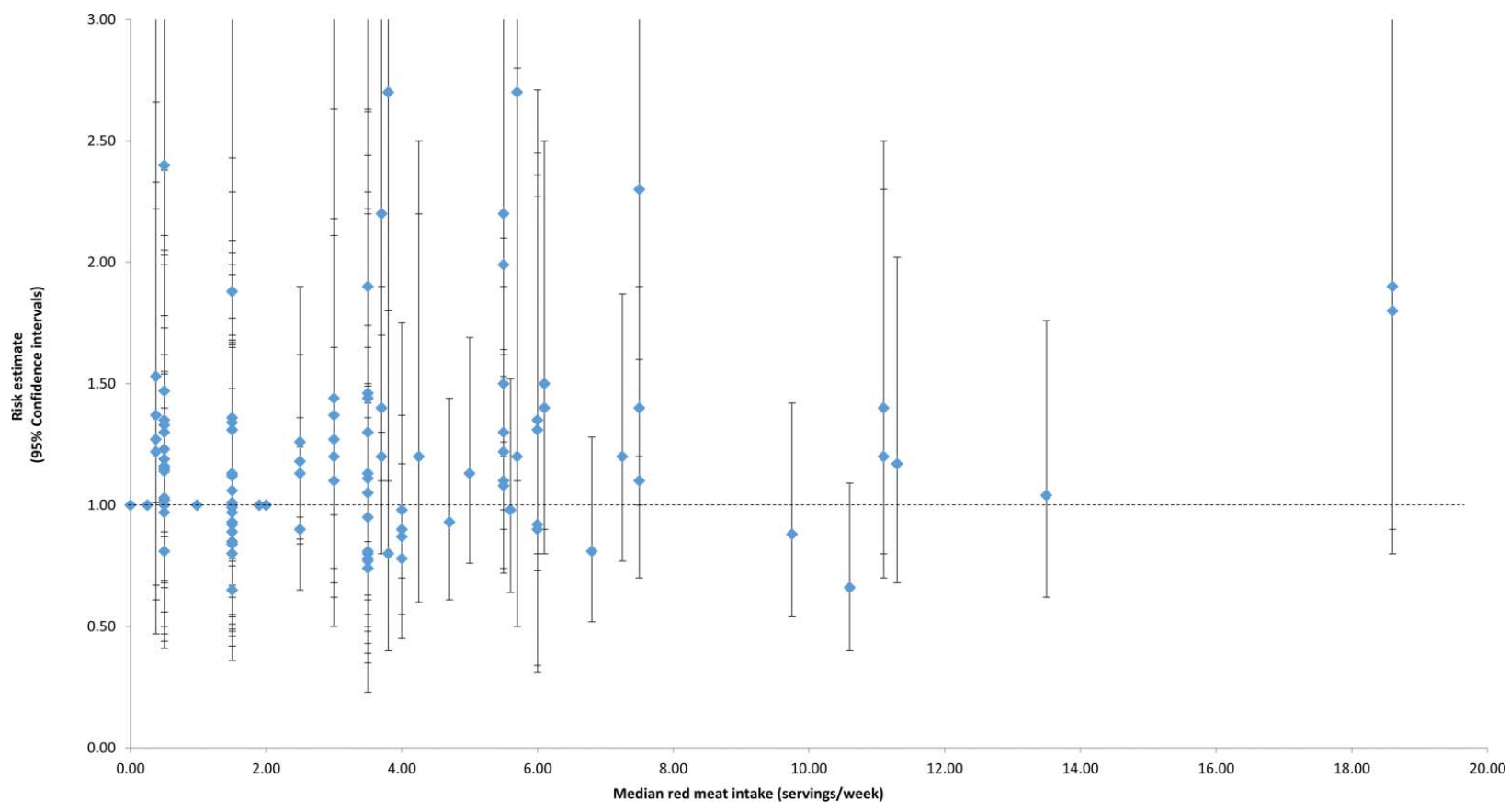

Fig. 3. Individual study relative risks for colorectal cancer across all red meat intake strata (servings / week) in the prospective cohort studies. 


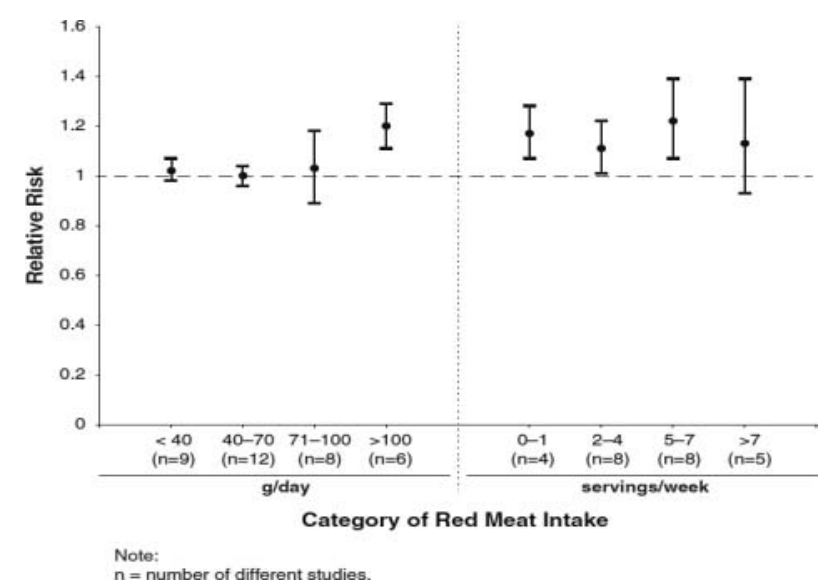

Fig. 4. Dose-response patterns based on meta-analyses of categorical intake groupings.

shown). The Duval and Tweedie trim-and-fill procedure imputed 3 studies to the left of the mean effect, resulting in an adjusted SRRE of 1.09 (95\% CI: 1.02-1.17), based on the allinclusive model.

\section{DISCUSSION}

In this updated and expanded meta-analysis, we observed summary associations for red meat and CRC that were null or just above the null value in virtually all models. Although some models were statistically significant, heterogeneity was present in many models, and findings varied by subgroups (e.g., SRRE for men $=1.16$; SRRE for women $=1.03$ ).

We attempted to isolate the red meat variable by identifying and analyzing studies that evaluated red meat but did not include processed meat items. Although there may be some overlap in these variables because some processed meat items often include red meat, and there is a high correlation between red meat intake and processed meat intake, summary associations were markedly reduced and not statistically significant when red meat only was evaluated.

Summary associations were slightly attenuated in our sensitivity analyses of studies that adjusted for relevant potential confounding factors, indicating the importance of measuring and adjusting for factors that may be related to both red meat consumption and CRC. Although we did not observe striking attenuation of the summary effect in models specific to confounding-factor adjustment, a meta-analysis may not be sensitive enough to fully appreciate the impact of confounding, especially if the epidemiological studies do not consistently control for the same confounding factors. The impact of confounding can best be discerned at the individual study level if the researchers report the crude (or age-adjusted only) RR and the multivariate RR. For example, in a recent prospective analysis, Ollberding et al. [43] reported an RR of 1.17 (95\% CI:
1.05-1.31) in the basic model (age and ethnicity), and an RR of 0.98 (95\% CI: $0.87-1.10$ ) in the multivariate model that adjusted for smoking, history of colorectal polyps, and BMI, among other factors. In fact, the majority of individual studies that report crude and adjusted RRs for red meat and CRC observe positive and stronger associations in the crude model compared to the more fully adjusted model. Even modest confounding and, perhaps just as importantly, slight measurement error in the ascertainment and classification of potential confounding variables can have a proportionally large impact on effect estimates [1,57].

The association between red meat intake and rectal cancer was slightly stronger than that for colon cancer, although both summary effects were attenuated in analyses of red meat only (without processed meat items included). It has been suggested that tumors arising in the proximal colon, distal colon, and rectum may have variable pathologies, and consequently, dietary factors may influence colorectal neoplasia differently according to anatomic site [58]. However, summary associations between colon and rectal tumor sites were not strikingly different. Although the literature is somewhat sparse, in general, findings for distal colon tumors are slightly stronger than for proximal colon tumors, which is anatomically consistent with the contiguous sites (i.e., proximal colon followed by distal colon followed by rectum).

Modification was also apparent by sex, with stronger effect sizes for men than women; however, summary associations were stronger for colon cancer than rectal cancer among men and stronger for rectal cancer than colon cancer among women (note that analyses were based on relatively limited data). Indeed, diet-related effects may differ by sex due to hormonal variation between men and women and by the proclivity of women to develop proximal tumors and men to develop distal and rectal tumors [58]. The reasons for the overall disparities in associations by gender are uncertain, and the differences in effect sizes do not appear to be the result of higher intake levels among men. In fact, when we grouped data by gender in the same intake categories, there were no discernible differences for the grams-per-day analyses, but there were considerable differences in the servings-per-week analyses. Summary associations were stronger for men for all serving categories compared with women, and these differences were not a function of higher consumption levels among men. Furthermore, there are no established biological or mechanistic differences that may have modified associations specifically for red meat intake. The stronger effects observed among men may be the result of stronger correlations with adverse dietary and lifestyle factors that further confound the association.

We conducted a comprehensive evaluation of relative risk point estimates reported in all prospective studies for all intake categories. Specifically, we used four methods to review the data (for both grams-per-day and servings-per-week metrics): (1) point estimates for all intake levels were plotted for a visual 


\section{Intake Level}

$10 \mathrm{~g} /$ day
$30 \mathrm{~g} /$ day
$50 \mathrm{~g} /$ day
$70 \mathrm{~g} /$ day
Range: $114-203$
$90 \mathrm{~g} /$ day

\section{Cumulative rate ratio $(95 \% \mathrm{CI})$}



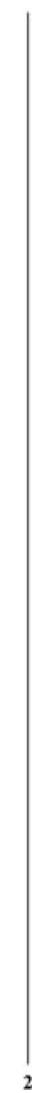

Fig. 5. Cumulative meta-analysis of colorectal cancer by grams/day of red meat intake.

assessment (Figs. 2 and 3), (2) categorical dose-response regression meta-analyses were conducted to estimate the linear (slope) effect by increasing categories of intake (Table 2), (3) meta-analyses for categorical intake groups were conducted to estimate associations for a more standardized evaluation (Table 2, Fig. 4), and (4) cumulative meta-analyses were performed to appreciate the modification and patterns of effect size by increasing dose levels (Figs. 5 and 6). If an exposure is associated with an outcome, it would be expected that consistent results would be observed across various dose-response analyses. However, for red meat consumption and CRC, no clear evidence of dose-response patterns emerges in a consistent fashion across the intake analyses. As described above, there are more point estimates above 1.0 than below, particularly for the servings-per-week metric, but there is no consistent pattern of increasing risk by increasing intake level (Figs. 2 and 3). The summary associations for the categorical dose-response regression analyses support only a weak positive association, but heterogeneity was present in these models. Further, this type of analysis assumes a linearly increasing pattern, which can be a problematic assumption if intake levels are highly variable among studies. In fact, our plots of RRs by specific intake levels were not consistent with a linear trend, and the categorical intake grouping analyses were inconsistent by metric and gender. No appreciable effect for red meat consumption and CRC was observed up to $100 \mathrm{~g} /$ day, but a modestly elevated SRRE of 1.20 (95\% CI: 1.11-1.29) was found for intake levels greater than $100 \mathrm{~g} /$ day. It is not clear if this association is a reflection of greater collinearity between other dietary and lifestyle factors at elevated levels of intake, or if higher intake levels of red meat may contribute to a slightly increased risk. However, this finding was not corroborated in the servings-per-week analyses, and no intake-response trend was apparent because considerable data inflection was observed (e.g., no monotonic pattern was evident). Moreover, as mentioned above, the elevations in the servings-per-week analyses were largely influenced by results among men. Finally, in our cumulative meta-analysis assessment of patterns of associations by increasing intake levels, no consistent trends of increasing summary effects were observed.

As expected, the summary associations were modified by study country, with slightly stronger (and more heterogeneous) associations among North American populations, with the weakest associations among Asian populations. The possible explanations for this observation are many; however, a Western dietary (and lifestyle) pattern is a likely explanation. Indeed, high intake of red meat has been correlated positively with factors that have been associated with increasing the risk 



Fig. 6. Cumulative meta-analysis of colorectal cancer by servings/week of red meat intake.

of CRC, such as a high BMI, smoking, and alcohol intake, and red meat intake has been correlated inversely with factors suggested as possibly decreasing the risk of CRC, such as physical activity, fruit and vegetable intake, and socioeconomic status. Thus, it is difficult to analytically isolate the separate effects of red meat from other dietary, lifestyle, socioeconomic, and clinical factors. Furthermore, multicollinearity plays a role here. Multicollinearity is a statistical phenomenon that occurs when two or more factors in a regression model are moderately to highly correlated, rendering the estimation of independent effects of a single dietary component difficult. Perhaps more so than other epidemiologic disciplines, nutritional epidemiology is prone to multicollinearity, because diet is a complex mixture of foods, nutrients, and other dietary constituents [1]. Greater consumption of one particular food tends to be associated with higher and lower intakes of other foods and nutrients that may also influence disease risk. Moreover, the influence of different dietary components may be multiplicative rather than purely additive, yet the ability to capture potential interactions of dietary components in statistical analyses may be limited. As a result, including highly correlated variables in the same statistical model may lead to biased risk estimates.

Of note, over time, there appears to be a time-dependent attenuation of summary associations-the earlier studies report stronger associations, while the more recent studies are generally closer to the null value, a phenomenon described by Ioannidis in an evaluation of clinical research studies [59]. Many potential factors may contribute to this observation; for example, recent studies may be adjusting for more relevant confounding factors, dietary instruments may have improved, and longer follow-up time has accrued in some cohorts that may allow for a more accurate reflection of cancer latency. If, however, the small association between red meat intake and CRC is real, the public health impact may be noteworthy because $\mathrm{CRC}$ is one of the more common malignancies.

Several postulated mechanisms by which consumption of red meat may contribute to colorectal carcinogenesis have been proposed. These include exposure to heterocyclic amines, polycyclic aromatic hydrocarbons, nitrate/nitrite and $\mathrm{N}$-nitroso compounds, fat intake from animal sources, changes to the intestinal microbiota, and heme iron. A thorough review of these compounds is beyond the scope of the current review, although the epidemiologic evidence regarding these hypothesized biological mechanisms has been inconsistent $[2,3,10,43,60-65]$. Of note, however, is the increased scientific focus on heme iron as a potential cancer promoter. It is important to distinguish between heme iron, which is from meat, and non-heme iron, which is from fortified cereals, fruit juice, and bread, among other sources [60]. In a recent review of heme iron from meat and CRC, a proposed mechanism of action was suggested whereby heme promotes ATNC (apparent total Nnitroso compounds) growth and lipid peroxidation production [66]. This may subsequently trigger activation of a series of steps on the biological pathway that facilitate DNA and 
cellular damage, which can eventually lead to adenocarcinoma [66]. Despite this theory, the epidemiologic evidence pertaining to heme iron and CRC has been weak and inconsistent. A summary relative risk of 1.18 (95\% CI: 1.06-1.32) was reported in a meta-analysis comparing high vs low heme iron intake and colon cancer [66]. However, recently published analyses were contradictory. In an analysis of the Nurses' Health Study and Health Professionals Follow-up Study, Zhang et al. [67] reported non-statistically significant inverse associations between heme iron and CRC among men, and nonsignificant positive associations among women. In contrast, Hara et al. [68] observed nonsignificant inverse associations among women and nonsignificant positive associations among men in an analysis of a population-based prospective cohort study in Japan. Diet plays a formative role in gut microbiota, and cancer risk may be modified by harmful and beneficial bacteria that could influence carcinogen bioactivation [2]. Currently, there is no clear evidence indicating that meat intake or animal protein consumption modifies CRC risk by altering the intestinal microflora. Clearly, more research is needed in this area, particularly because the associations between red meat consumption and CRC across the epidemiologic studies have been weak and relatively inconsistent.

Complicating the interpretation of findings from nutritional epidemiology studies is the fact that this research area is particularly prone to reporting bias because of the numerous types of foods, food combinations, nutrients, and cooking methods ascertained on a typical food frequency questionnaire (FFQ). Upward of 150 food items (in addition to serving size and cooking practices, among other things) are commonly queried on an FFQ; thus, a researcher may selectively choose which data to report when analyzing all foods. If, for example, red meat, or any other food item for that matter, is not associated with cancer in an analysis of numerous food items, the researcher may selectively withhold the "null" results and choose to submit for publication the non-null, or the perceived more impactful, findings. Furthermore, in the absence of an observed association for which an existing relationship was assumed, a researcher may withhold the results, because they are not in line with the prevailing scientific opinion, whether accurate or not.

Although meta-analysis methods are being used at an increasing rate to quantitatively summarize a body of literature, meta-analyses may suffer from the same shortcomings and challenges that influence the individual studies. In fact, a metaanalysis may actually amplify the inherent limitations from individual studies because of combining data across studies that may be biased or confounded to produce an overall effect. Combining data at the macro level does not obviate the complications of interpreting the data on a micro level. However, given a large volume of literature, a meta-analysis can be used to create informative subgroup stratifications, to examine potential sources of heterogeneity and to evaluate the consistency of results by partially controlling for some of the study-specific characteristics. In addition, a meta-analysis improves the precision of summary estimates of effect, which is especially important when attempting to demonstrate patterns of associations across subgroups. Important insights into some (but certainly not all) of the most important aspects of causation can be provided by a meta-analysis, and it has the capacity to better characterize the existence and nature of associations summarized across studies [3]. It is generally well established that a causal relationship is more likely when the association is strong (typically relative risks above 2.0), there is a clear dose-response effect (i.e., increasing risk with increasing exposure), and results are consistent (e.g., across studies, study designs, and similar study characteristics). In our study, all summary associations between red meat consumption and CRC were weak in magnitude, no coherent pattern of doseresponse was apparent, and associations were largely inconsistent across the individual studies and the analytical subgroups. Such patterns of association are not supportive of a relationship that is causal. Furthermore, associations from human health studies of the postulated biological mechanisms have been weak and inconsistent as well.

Until the methodological and analytical approach to evaluate red meat and CRC is modified or a new approach is introduced (such as creating multiple layers of subgroup stratifications by presumably important factors), each subsequent analysis of a cohort will likely keep producing associations that hover around the null value. Clinical trials of a food or food group and cancer are not common (none exist for red meat and cancer), because of some obvious challenges, such as cost and long duration to account for cancer latency. Although theoretically possible, it is not likely that such a study will be conducted (and with sufficient follow-up time) in the near future. As a result, researchers will need to analyze data from new cohorts and continue to publish updated and extended analyses on existing cohorts.

In the current meta-analysis of red meat intake and CRC, we comprehensively examined associations by creating numerous subgroup stratifications, conducting extensive sensitivity analyses, and evaluating dose-response using several different methods. Associations between red meat and CRC were weak to nonexistent. Of the highest categories of intake in the individual studies, over one-third of the RRs were 1.0 or lower, and almost half were less than 1.05. Summary associations were weak, with most SRREs around the null value or just slightly above. Because of multiple comparisons (i.e., over 70 separate analyses were conducted), some statistically significant associations could arise by chance alone. Indeed, we did not observe consistent patterns of associations that may explain the few significant summary associations. Even in studies that analyzed red meat independent of processed meat, and adjusted for additional relevant confounding factors, models found summary associations to be attenuated, though slightly less 
heterogeneous. Associations were stronger among men than women, and stronger for rectal cancer than for colon cancer, but within each sex, the results were contrasted by tumor site, which observation is yet another example of inconsistency. Summary associations are also likely influenced by dietary and lifestyle factors; a case in point is that studies of American cohorts showed stronger associations than studies of cohorts who do not have a Western diet and lifestyle. Finally, summary associations appear to be weakening over time, because the SRREs in the more recent studies are weaker and closer to the null value than those of earlier studies.

Methodological limitations and challenges compromise the ability to interpret a possible relationship between red meat and CRC. Indeed, given the overall lack of consistent findings, and the absence of strong associations and dose-response patterns, it seems reasonable to posit that red meat is a negligible factor in CRC risk; rather, there is a more significant constellation of dietary and lifestyle factors that may influence colorectal carcinogenesis. The quantitative findings and scientific rationale for interpretation documented in the current metaanalysis support a conclusion that red meat is not an independent predictor of CRC risk.

In conclusion, based on the quantitative findings and scientific rationale for interpretation documented in the current meta-analysis, red meat intake does not appear to be an independent predictor of CRC risk.

\section{FUNDING}

This manuscript was partially funded by Meat \& Livestock Australia (MLA). MLA did not contribute to the writing, analysis, or interpretation of study findings.

\section{REFERENCES}

1. Miller PE, Alexander DD, Weed DL: Uncertainty of results in nutritional epidemiology. Nutrition Today 49:147-152, 2014.

2. Kim E, Coelho D, Blachier F: Review of the association between meat consumption and risk of colorectal cancer. Nutr Res 33:983994, 2013.

3. Alexander DD, Weed DL, Cushing CA, Lowe KA: Meta-analysis of prospective studies of red meat consumption and colorectal cancer. Eur J Cancer Prev 20:293-307, 2011.

4. Smolinska K, Paluszkiewicz P: Risk of colorectal cancer in relation to frequency and total amount of red meat consumption. Systematic review and meta-analysis. Arch Med Sci 6:605-610, 2010.

5. Alexander DD, Weed DL, Chang ET, Miller PE, Mohamed MA, Elkayam L: A systematic review of multivitamin-multimineral use and cardiovascular disease and cancer incidence and total mortality. J Am Coll Nutr 32:339-354, 2013.

6. Chan DS, Lau R, Aune D, Vieira R, Greenwood DC, Kampman E, Norat T: Red and processed meat and colorectal cancer incidence: Meta-analysis of prospective studies. PLoS One 6:e20456, 2011.
7. Larsson SC, Wolk A: Meat consumption and risk of colorectal cancer: A meta-analysis of prospective studies. Int J Cancer 119:2657-2664, 2006.

8. Norat T, Bingham S, Ferrari P, Slimani N, Jenab M, Mazuir M, Overvad K, Olsen A, Tjonneland A, Clavel F, Boutron-Ruault MC, Kesse E, Boeing H, Bergmann MM, Nieters A, Linseisen J, Trichopoulou A, Trichopoulos D, Tountas Y, Berrino F, Palli D, Panico S, Tumino R, Vineis P, Bueno-de-Mesquita HB, Peeters PH, Engeset D, Lund E, Skeie G, Ardanaz E, Gonzalez C, Navarro C, Quiros JR, Sanchez MJ, Berglund G, Mattisson I, Hallmans G, Palmqvist R, Day NE, Khaw KT, Key TJ, San JM, Hemon B, Saracci R, Kaaks R, Riboli E: Meat, fish, and colorectal cancer risk: The European Prospective Investigation into cancer and nutrition. J Natl Cancer Inst 97:906-916, 2005.

9. Sandhu MS, White IR, McPherson K: Systematic review of the prospective cohort studies on meat consumption and colorectal cancer risk: A meta-analytical approach. Cancer Epidemiol Biomarkers Prev 10:439-446, 2001.

10. Alexander DD, Cushing CA: Red meat and colorectal cancer: A critical summary of prospective epidemiologic studies. Obes Rev 12:e472-e493, 2011.

11. World Cancer Research Fund/American Institute for Cancer Research. Food, nutrition, physical activity, and the prevention of cancer: A global perspective. 1-517. 2007. Washington, D.C., American Institute for Cancer Research (AICR).

12. Warriss PD: "Meat Science: An Introductory Text. Wallingford, Oxon, UK: CABI Publishing, 2000.

13. Alexander DD, Miller AJ, Cushing CA, Lowe KA: Processed meat and colorectal cancer: A quantitative review of prospective epidemiologic studies. Eur J Cancer Prev 19:328-341, 2010.

14. Alexander DD, Cushing CA, Lowe KA, Sceurman B, Roberts MA: Meta-analysis of animal fat or animal protein intake and colorectal cancer. Am J Clin Nutr 89:1402-1409, 2009.

15. Bostick RM, Potter JD, Kushi LH, Sellers TA, Steinmetz KA, McKenzie DR, Gapstur SM, Folsom AR: Sugar, meat, and fat intake, and non-dietary risk factors for colon cancer incidence in Iowa women (United States). Cancer Causes Control 5:38-52, 1994.

16. Takachi R, Tsubono Y, Baba K, Inoue M, Sasazuki S, Iwasaki M, Tsugane S, Japan Public Health Center-Based Prospective Study Group: Red meat intake may increase the risk of colon cancer in Japanese, a population with relatively low red meat consumption. Asia Pac J Clin Nutr 20:603-612, 2011.

17. Brink M, Weijenberg MP, de Goeij AF, Roemen GM, Lentjes MH, de Bruine AP, Goldbohm RA, van den Brandt PA: Meat consumption and K-ras mutations in sporadic colon and rectal cancer in The Netherlands Cohort Study. Br J Cancer 92:1310-1320, 2005.

18. Butler LM, Wang R, Koh WP, Yu MC: Prospective study of dietary patterns and colorectal cancer among Singapore Chinese. Br J Cancer 99:1511-1516, 2008.

19. Chan AT, Tranah GJ, Giovannucci EL, Willett WC, Hunter DJ, Fuchs CS: Prospective study of N-acetyltransferase-2 genotypes, meat intake, smoking and risk of colorectal cancer. Int J Cancer 115:648-652, 2005.

20. Chao A, Thun MJ, Connell CJ, McCullough ML, Jacobs EJ, Flanders WD, Rodriguez C, Sinha R, Calle EE: Meat consumption and risk of colorectal cancer. JAMA 293:172-182, 2005. 
21. Chen J, Stampfer MJ, Hough HL, Garcia-Closas M, Willett WC, Hennekens CH, Kelsey KT, Hunter DJ: A prospective study of Nacetyltransferase genotype, red meat intake, and risk of colorectal cancer. Cancer Res 58:3307-3311, 1998.

22. Chen K, Cai J, Liu XY, Ma XY, Yao KY, Zheng S: Nested casecontrol study on the risk factors of colorectal cancer. World J Gastroenterol 9:99-103, 2003.

23. Cross AJ, Leitzmann MF, Gail MH, Hollenbeck AR, Schatzkin A, Sinha R: A prospective study of red and processed meat intake in relation to cancer risk. PLoS Med 4:e325, 2007.

24. English DR, MacInnis RJ, Hodge AM, Hopper JL, Haydon AM, Giles GG: Red meat, chicken, and fish consumption and risk of colorectal cancer. Cancer Epidemiol Biomarkers Prev 13:1509_ 1514, 2004.

25. Flood A, Velie EM, Sinha R, Chaterjee N, Lacey JV, Jr., Schairer C, Schatzkin A: Meat, fat, and their subtypes as risk factors for colorectal cancer in a prospective cohort of women. Am J Epidemiol 158:59-68, 2003.

26. Fraser GE: Associations between diet and cancer, ischemic heart disease, and all-cause mortality in non-Hispanic white California Seventh-day Adventists. Am J Clin Nutr 70:532S-538S, 1999.

27. Fung TT, Hu FB, Wu K, Chiuve SE, Fuchs CS, Giovannucci E: The Mediterranean and Dietary Approaches to Stop Hypertension (DASH) diets and colorectal cancer. Am J Clin Nutr 92:14291435,2010

28. Gaard M, Tretli S, Loken EB: Dietary factors and risk of colon cancer: A prospective study of 50,535 young Norwegian men and women. Eur J Cancer Prev 5:445-454, 1996.

29. Giovannucci E, Rimm EB, Stampfer MJ, Colditz GA, Ascherio A, Willett WC: Intake of fat, meat, and fiber in relation to risk of colon cancer in men. Cancer Res 54:2390-2397, 1994.

30. Hsing AW, McLaughlin JK, Chow WH, Schuman LM, Co Chien HT, Gridley G, Bjelke E, Wacholder S, Blot WJ: Risk factors for colorectal cancer in a prospective study among U.S. white men. Int J Cancer 77:549-553, 1998.

31. Iso H, Kubota Y: Nutrition and disease in the Japan Collaborative Cohort Study for Evaluation of Cancer (JACC). Asian Pac J Cancer Prev 8 Suppl:35-80.:35-80, 2007.

32. Jarvinen R, Knekt P, Hakulinen T, Rissanen H, Heliovaara M: Dietary fat, cholesterol and colorectal cancer in a prospective study. Br J Cancer 85:357-361, 2001.

33. Kabat GC, Miller AB, Jain M, Rohan TE: A cohort study of dietary iron and heme iron intake and risk of colorectal cancer in women. Br J Cancer 97:118-122, 2007.

34. Kato I, Akhmedkhanov A, Koenig K, Toniolo PG, Shore RE, Riboli E: Prospective study of diet and female colorectal cancer: The New York University Women's Health Study. Nutr Cancer 28:276-281, 1997.

35. Khan MM, Goto R, Kobayashi K, Suzumura S, Nagata Y, Sonoda T, Sakauchi F, Washio M, Mori M: Dietary habits and cancer mortality among middle aged and older Japanese living in hokkaido, Japan by cancer site and sex. Asian Pac J Cancer Prev 5:58-65, 2004.

36. Kojima M, Wakai K, Tamakoshi K, Tokudome S, Toyoshima H, Watanabe Y, Hayakawa N, Suzuki K, Hashimoto, Ito Y, Tamakoshi A: Diet and colorectal cancer mortality: Results from the Japan Collaborative Cohort Study. Nutr Cancer 50:23-32, 2004.
37. Larsson SC, Rafter J, Holmberg L, Bergkvist L, Wolk A: Red meat consumption and risk of cancers of the proximal colon, distal colon and rectum: The Swedish Mammography Cohort. Int J Cancer 20;113:829-834, 2005.

38. Lee SA, Shu XO, Yang G, Li H, Gao YT, Zheng W: Animal origin foods and colorectal cancer risk: A report from the Shanghai Women's Health Study. Nutr Cancer 61:194-205, 2009.

39. Lin J, Zhang SM, Cook NR, Lee IM, Buring JE: Dietary fat and fatty acids and risk of colorectal cancer in women. Am J Epidemiol 160:1011-1022, 2004.

40. Luchtenborg M, Weijenberg MP, de Goeij AF, Wark PA, Brink M, Roemen GM, Lentjes MH, de Bruine AP, Goldbohm RA, van,'t Veer P, van den Brandt PA: Meat and fish consumption, APC gene mutations and hMLH1 expression in colon and rectal cancer: A prospective cohort study (The Netherlands). Cancer Causes Control 16:1041-1054, 2005.

41. Nothlings U, Yamamoto JF, Wilkens LR, Murphy SP, Park SY, Henderson BE, Kolonel LN, Le ML: Meat and heterocyclic amine intake, smoking, NAT1 and NAT2 polymorphisms, and colorectal cancer risk in the multiethnic cohort study. Cancer Epidemiol Biomarkers Prev 18:2098-2106, 2009.

42. Oba S, Shimizu N, Nagata C, Shimizu H, Kametani M, Takeyama $\mathrm{N}$, Ohnuma T, Matsushita S: The relationship between the consumption of meat, fat, and coffee and the risk of colon cancer: A prospective study in Japan. Cancer Lett 244:260-267, 2006.

43. Ollberding NJ, Wilkens LR, Henderson BE, Kolonel LN, Le ML: Meat consumption, heterocyclic amines and colorectal cancer risk: The Multiethnic Cohort Study. Int J Cancer 131:E1125-E1133, 2012.

44. Pietinen P, Malila N, Virtanen M, Hartman TJ, Tangrea JA, Albanes D, Virtamo J: Diet and risk of colorectal cancer in a cohort of Finnish men. Cancer Causes Control 10:387-396, 1999.

45. Sato Y, Nakaya N, Kuriyama S, Nishino Y, Tsubono Y, Tsuji I: Meat consumption and risk of colorectal cancer in Japan: The Miyagi Cohort Study. Eur J Cancer Prev 15:211-218, 2006.

46. Sellers TA, Bazyk AE, Bostick RM, Kushi LH, Olson JE, Anderson KE, Lazovich D, Folsom AR: Diet and risk of colon cancer in a large prospective study of older women: An analysis stratified on family history (Iowa, United States). Cancer Causes Control 9:357-367, 1998.

47. Singh PN, Fraser GE: Dietary risk factors for colon cancer in a low-risk population. Am J Epidemiol 148:761-774, 1998.

48. Thun MJ, Calle EE, Namboodiri MM, Flanders WD, Coates RJ, Byers T, Boffetta P, Garfinkel L, Heath CW, Jr.: Risk factors for fatal colon cancer in a large prospective study. J Natl Cancer Inst 84:1491-1500, 1992.

49. Tiemersma EW, Kampman E, Bueno de Mesquita HB, Bunschoten A, van Schothorst EM, Kok FJ, Kromhout D: Meat consumption, cigarette smoking, and genetic susceptibility in the etiology of colorectal cancer: Results from a Dutch prospective study. Cancer Causes Control 13:383-393, 2002.

50. Wei EK, Giovannucci E, Wu K, Rosner B, Fuchs CS, Willett WC, Colditz GA: Comparison of risk factors for colon and rectal cancer. Int J Cancer 108:433-442, 2004.

51. Willett WC, Stampfer MJ, Colditz GA, Rosner BA, Speizer FE: Relation of meat, fat, and fiber intake to the risk of colon cancer in a prospective study among women. N Engl J Med 323:1664$1672,1990$. 
52. Wu K, Hu FB, Fuchs C, Rimm EB, Willett WC, Giovannucci E: Dietary patterns and risk of colon cancer and adenoma in a cohort of men (United States). Cancer Causes Control 15:853-862, 2004.

53. Greenland S, Longnecker MP: Methods for trend estimation from summarized dose-response data, with applications to meta-analysis. Amer J Epid 135:1301-1309, 1992.

54. Berlin JA, Longnecker MP, Greenland S: Meta-analysis of epidemiologic dose-response data. Epidemiology 4:218-228, 1993.

55. Orsini N, Li R, Wolk A, Khudyakov P, Spiegelman D: Meta-analysis for linear and nonlinear dose-response relations: examples, an evaluation of approximations, and software. Am J Epidemiol 175:66-73, 2012.

56. Rothstein HR, Sutton AJ, Borenstein M: "Publication Bias in Meta-Analysis: Prevention, Assessment, and Adjustments." Chichester, West Sussex, UK: John Wiley \& Sons Ltd., 2005.

57. Schatzkin A, Kipnis V: Could exposure assessment problems give us wrong answers to nutrition and cancer questions? J Natl Cancer Inst 96:1564-1565, 2004.

58. Jacobs ET, Thompson PA, Martinez ME: Diet, gender, and colorectal neoplasia. J Clin Gastroenterol 41:731-746, 2007.

59. Ioannidis JP: Contradicted and initially stronger effects in highly cited clinical research. JAMA 294:218-228, 2005.

60. Cross AJ, Ferrucci LM, Risch A, Graubard BI, Ward MH, Park Y, Hollenbeck AR, Schatzkin A, Sinha R: A large prospective study of meat consumption and colorectal cancer risk: an investigation of potential mechanisms underlying this association. Cancer Res 70:2406-2414, 2010.

61. Cross AJ, Harnly JM, Ferrucci LM, Risch A, Mayne ST, Sinha R: Developing a heme iron database for meats according to meat type, cooking method and doneness level. Food Nutr Sci 3:905913, 2012.

62. Butler LM, Sinha R, Millikan RC, Martin CF, Newman B, Gammon MD, Ammerman AS, Sandler RS: Heterocyclic amines, meat intake, and association with colon cancer in a population-based study. Am J Epidemiol 157:434-445, 2003.

63. Sinha R, Peters U, Cross AJ, Kulldorff M, Weissfeld JL, Pinsky PF, Rothman N, Hayes RB: Meat, meat cooking methods and preservation, and risk for colorectal adenoma. Cancer Res 65:8034-8041, 2005.

64. Augustsson K, Skog K, Jagerstad M, Dickman PW, Steineck G: Dietary heterocyclic amines and cancer of the colon, rectum, bladder, and kidney: A population-based study. Lancet 353:703-707, 1999.

65. Santarelli RL, Pierre F, Corpet DE: Processed meat and colorectal cancer: A review of epidemiologic and experimental evidence. Nutr Cancer 60:131-144, 2008.

66. Bastide NM, Pierre FH, Corpet DE: Heme iron from meat and risk of colorectal cancer: A meta-analysis and a review of the mechanisms involved. Cancer Prev Res (Phila) 4:177-184, 2011.

67. Zhang X, Giovannucci EL, Smith-Warner SA, Wu K, Fuchs CS, Pollak M, Willett WC, Ma J: A prospective study of intakes of zinc and heme iron and colorectal cancer risk in men and women. Cancer Causes Control 22:1627-1637, 2011.

68. Hara A, Sasazuki S, Inoue M, Iwasaki M, Shimazu T, Sawada N, Yamaji T, Takachi R, Tsugane S: Zinc and heme iron intakes and risk of colorectal cancer: A population-based prospective cohort study in Japan. Am J Clin Nutr 96:864-873, 2012.

Received September 18, 2014; accepted November 24, 2014. 